\title{
Colloids dispersed in polymer solutions. A computer simulation study
}

\author{
Evert Jan Meijer and Daan Frenkel \\ FOM Institute for Atomic and Molecular Physics, Kruislaan 407, 1098 SJ Amsterdam, The Netherlands
}

(Received 15 December 1993; accepted 27 January 1994)

\begin{abstract}
We report a computer-simulation study of a simple model for a colloid dispersed in a polymer solution. The simulations were performed using a computational scheme that allows simulation at constant osmotic pressure of the polymers. We present results for the polymer-induced interaction, the equation-of-state, and the phase diagram. The simulations show that when the radius of gyration of the polymers $R_{g}$ is sufficiently large compared to the diameter of the colloidal particles $\sigma_{\text {col }}$ $\left(2 R_{g} / \sigma_{\text {col }} \geqslant 0.45\right)$, addition of polymer induces a colloidal "liquid-vapor" phase separation. For shorter polymers, only the solid-fluid transition is observed. In addition, we find that the nonpairwise additivity of the polymer-induced attraction between the colloidal particles has a pronounced effect on the equation-of-state and the phase behavior of a colloid-polymer mixture. The perturbation theory of Lekkerkerker et al. [Europhys. Lett. 20, 559 (1992)] is found to perform well, except at low densities.
\end{abstract}

\section{INTRODUCTION}

Adding nonadsorbing polymer to a colloidal dispersion gives rise to a change in the properties of the dispersion. The main effect is that, upon adding enough polymer, the colloidal particles tend to cluster. This effect was first reported some 50 years ago. ${ }^{1,2}$ A theoretical explanation was first put forward by Asakura and Oosawa ${ }^{3}$ and later in more detail by Vrij. ${ }^{4}$ These authors argued that the clustering of the colloidal particles is due to the fact that the presence of the polymers induces an effective attractive interaction between the colloidal particles. This polymer-induced attraction stems from the fact that clustering of the colloid increases the volume accessible to the polymers.

Several experimental studies of the effect of adding polymer to a colloidal dispersion have been reported. ${ }^{5-16}$ These studies were concerned with the dependence of ordering phenomena in the colloid on the polymer concentration, on the type of solvent, and on the polymer length. A feature observed in most of these experimental studies is the aggregation of colloidal particles upon the addition of polymer. The colloid-polymer mixtures may exhibit properties analogous to those of the vapor, liquid, or solid phases of simple molecular systems. The experiments indicate that the type of phase that appears depends on the polymer-colloid size ratio $R_{g} / \sigma_{\text {col }}$, where $R_{g}$ denotes the radius-of-gyration of the polymers and $\sigma_{\text {col }}$ the diameter of the colloidal particles. For example, for a colloid dispersed in a nonaqueous medium, ${ }^{6.8 .14 .16}$ it was found that upon adding polymer, a dilute colloidal "fluid"-like phase separates in a dilute "vapor"-like phase and a dense "liquid"- or "solid"-like phase. The "liquid"-like phase was reported to occur for polymers with relatively large size ratio $\left(2 R_{g} / \sigma_{\text {col }} \sim 1\right)$ and was absent for shorter polymers $\left(2 R_{g} / \sigma_{\mathrm{col}} \sim 0.1\right) .{ }^{14}$ More recently, a similar behavior has been reported for a colloid dispersed in an aqueous dispersion. ${ }^{17}$

The theoretical study of the structural properties and phase equilibria of colloid-polymer mixtures was initiated by $V_{r i j}{ }^{4}$ who used a simple model, introduced earlier by Asakura and Oosawa. ${ }^{3}$ In the Asakura-Oosawa (AO) model the colloidal particles are represented by hard spheres, and the polymers by spheres that are mutually interpenetrable but have an excluded-volume interaction with the colloidal hard spheres. Various refinements of the AO-model have been proposed taking into account the deformability of the polymers and the interactions among the polymer molecules. Some of these theories employ mean-field theory, ${ }^{14,18}$ while others are based on scaling arguments. ${ }^{19,20}$ In either case, it is usually assumed that the radius-of-gyration of the polymer is much smaller than the radius of the colloidal particles. Some authors have considered the AO-model with a modified interaction potential for the colloidal particles, ${ }^{13,20,21}$ in order to adapt the model to specific experimental systems. Finally, a few theoretical studies of colloid-polymer mixtures are based on integral equations. ${ }^{22,23}$

In most theoretical studies, the colloid-polymer mixtures are described as one-component systems, where the contribution of the polymers is replaced by an effective polymer-induced interaction, added to the bare colloidal interaction potential. The polymer-induced interaction, that is (for most models) directly related to the volume accessible to the polymers, is not pairwise additive in the coordinates of the colloidal particles. Only for the smallest polymers, i.e., those with a radius-of-gyration much smaller than the radius of the colloidal spheres, a pair-approximation is expected to be applicable. ${ }^{24,25}$ However, in view of the difficulties encountered in solving models with nonpairwise additive interactions, most theoretical approaches assume the polymerinduced interaction to be pairwise additive. Only recently a study has been reported ${ }^{26,27}$ that takes into account the nonpairwise additivity of the polymer-induced interaction, by applying scaled particle theory. Methods used to compute the thermodynamic properties and the phase behavior include thermodynamic perturbation theory ${ }^{13,20,24,26}$ and variation calculus. $^{21}$ The location of phase-coexistence curves has been predicted for various values of the polymer-colloid size ratio $R_{g} / \sigma_{\text {col }}$. The common trend of these theoretical results is that for short polymers the phase diagram is of the fluid-solid type, whereas for longer polymers it exhibits a gas-liquid phase separation of the fluid phase. This is quali- 
tatively in accordance with the experimental observations.

Still, the theoretical models for colloid-polymer mixtures remain too complex to be solved exactly, and one is forced to make drastic, and often untested, approximations. It is therefore desirable to know the "exact" properties of model colloid-polymer mixtures. These can be provided by computer simulation. Numerical simulation of microscopic models for colloids dispersed in a polymer solution would require orders of magnitude more computer time than simulations of similar models for atomic or simple molecular systems. The reason is twofold. First, the relaxation times in mixtures are, in general, longer than in pure systems. Second, the relaxation times in polymeric systems are orders of magnitude longer than in atomic systems. Consequently, one has to perform longer simulations to sample a representative part of the phase space. Fortunately, many of the properties of colloid-polymer mixtures do not depend sensitively on the molecular details of the model. In the present paper we present results of an extensive simulation study of a simple lattice-polymer model, introduced earlier. ${ }^{25}$ In this model, the colloids are represented by hard spheres and the polymers by ideal lattice chains. Although the lattice-polymer model is clearly too simple to be a realistic, it includes the essential elements; it takes into account the nonpairwise additivity of the polymer-induced interaction, and, unlike the AO-model, it takes into account the deformability of the polymer coils. For comparison we also present simulation results for the $\mathrm{AO}$-model.

The "exact" properties of the model colloid-polymer mixtures obtained by computer simulations provide can be used to test the validity of approximations made in theoretical descriptions of these systems. In this work we focus on the approximations made in thermodynamic perturbation theory and scaled-particle theory, that are among the theories most often applied, and the assumption of a pairwise additive polymer-induced interaction.

The present paper is organized as follows. In Sec. II, we describe the models considered, and formulate the thermodynamic properties starting from a statistical mechanical description. We briefly discuss thermodynamic perturbation theory and scaled particle theory. The numerical techniques used to study the models are presented in Secs. III A and III B. Next (Secs. IV and V), we present results obtained for the polymer-induced interaction and thermodynamic properties, including the phase behavior.

The results of the simulations are compared with the relevant theoretical predictions (Scc. VI). In particular, we investigate the accuracy of scaled-particle theory and thermodynamic perturbation theory, and we test the validity of the Asakura-Oosawa model in which the deformability of the polymers coils is neglected. This comparison indicates that it is usually not permissible to neglect the many-body character of the polymer-induced interactions. The perturbation theory of Lekkerkerker et al. ${ }^{26}$ is found to perform well at high colloid densities, but less so in the vicinity of the liquid-vapor critical point. Section VII provides a summary of the results of the present paper and indicates problems that should be addressed in future work.

\section{MODEL AND THERMODYNAMICS}

In this work we consider two models for a colloid dispersed in a dilute polymer solution, namely a latticepolymer model ${ }^{25}$ and the Asakura-Oosawa (AO) model. ${ }^{3,4}$ In both models the colloidal particles are represented by hard spheres with a diameter denoted by $\sigma_{\text {col }}$. In the latticepolymer model the polymers are represented by ideal (nonself-avoiding) chains that are confined to a cubic lattice. The length of the polymer, i.e., the number of connected lattice links that constitute the polymer, is denoted by $l$. If every occupied lattice site is associated with a polymer segment, an $l$-link lattice polymer has $l+1$ segments. The solvent is not taken into account explicitly. The ideal polymers have an excluded-volume interaction with the colloidal hard spheres; the lattice sites occupied by the colloidal hard spheres are not accessible to the polymer segments. The lattice sites not occupied by the colloidal hard spheres can be occupied by all polymer segments with equal probability. Although quite simple, the model is expected to yield a fair description of a subclass of the systems studied experimentally (see, e.g., Refs. 6,8,14,16). For example, the interaction between silica particles in an organic solvent is very close to a hard-sphere interaction. Dilute solutions of polymers in a theta-solvent are reasonably well modeled by noninteracting polymers. In the AO model the polymers are represented by ideal spheres with diameter $\sigma_{p o l}$. In this work we assume that the positions of the polymer spheres are restricted to a cubic lattice.

In the following we express all distances in units of the lattice spacing. Free energies will be expressed in units of $k_{B} T$, where $k_{B}$ denotes the Boltzmann constant, and $T$ the temperature. In the remainder of this section we refer mainly to the lattice-polymer model. The discussion of the $\mathrm{AO}$ model is analogous.

Consider a system of $N$ colloidal hard spheres in a volume $V$ in chemical equilibrium with an large reservoir of ideal lattice chains with fugacity $z$. For ideal polymers, the fugacity is proportional to the osmotic pressure $\Pi$ and related to chemical potential $\mu_{\text {pol }}$ by $z=\exp \left(\mu_{\text {pol }}\right)$. As the polymers are ideal, the condition of constant polymer chemical potential is a convenient choice. For a given colloid configuration $\mathbf{r}^{N}$ the partition function of a single lattice polymer is given by the total number of accessible conformations $\Omega\left(\mathbf{r}^{N} ; V\right)$ (for the AO-model this would be the accessible or free volume). As the polymers are ideal the partition function for a system with $M$ polymers is given by

$$
\frac{\left[\Omega\left(\mathbf{r}^{N} ; V\right)\right]^{M}}{M !}
$$

This allows us to write the partition function of the colloidpolymer mixture at constant $(N, z, V)$ as, ${ }^{25}$ 


$$
\begin{aligned}
\Xi(N, z, V)= & \int_{V} d \mathbf{r}^{N} \exp \left[-U_{\mathrm{hs}}\left(\mathbf{r}^{N}\right)\right] \\
& \times \sum_{M=0}^{\infty} \exp \left(M \mu_{\mathrm{pol}}\right) \frac{\left[\Omega\left(\mathbf{r}^{N} ; V\right)\right]^{M}}{M !} \\
= & \int_{V} d \mathbf{r}^{N} \exp \left[-U_{\mathrm{hs}}\left(\mathbf{r}^{N}\right)+z \Omega\left(\mathbf{r}^{N} ; V\right)\right] .
\end{aligned}
$$

Here, $U_{\text {hs }}$ denotes the hard-sphere interaction between the colloidal particles. Equation (1) shows that the $\Xi$ can be interpreted as the canonical partition function for a onecomponent system with an interaction potential given by

$$
U_{\text {col }+ \text { pol }}\left(\mathbf{r}^{N} ; V\right)=U_{\text {hs }}\left(\mathbf{r}^{N}\right)-z \Omega\left(\mathbf{r}^{N} ; V\right),
$$

where the product of the fugacity and the one-polymer partition function $-z \Omega$ is identified as the polymer-induced interaction.

A convenient expression for the polymer-induced interaction is obtained when it is expressed in terms of the total number of excluded conformations $\vec{\Omega}$, defined as

$$
\Omega\left(\mathbf{r}^{N} ; V\right)=\Omega^{i d}(V)-\bar{\Omega}\left(\mathbf{r}^{N}\right)
$$

Here the ideal term, $\Omega^{\text {id }}(V)$ denotes the number of conformations on an empty cubic lattice,

$$
\Omega^{\text {id }}(V)=\frac{1}{2} V \sigma^{l} \text {. }
$$

In terms of $\bar{\Omega}$, the effective interaction potential for the colloid-polymer mixture [Eq. (2)] can be written as

$$
U_{\text {col }+ \text { pol }}\left(\mathbf{r}^{N}\right)=U_{\mathrm{hs}}\left(\mathbf{r}^{N}\right)+z \bar{\Omega}\left(\mathbf{r}^{N}\right) \text {, }
$$

where we omitted the ideal term $-z \Omega^{\text {id }}$ as it does not contribute to the thermodynamic properties.

Clearly, the polymer-induced interaction $z \bar{\Omega}$ provides an attractive contribution. Upon decreasing the distance between the colloidal particles, the number of excluded conformations $\bar{\Omega}$ decreases due to an increasing number of conformations excluded by the two or more colloidal particles simultaneously. This interaction is known as the depletion interaction, and is purely entropic. The total number of excluded polymer conformations $\bar{\Omega}$ is not pairwise additive in the colloidal coordinates $\mathbf{r}^{N}$. In order to quantify the manybody character of $\bar{\Omega}$, we write it as an expansion of one-, two-, three-, and more-body terms. For two colloidal particles the total number of excluded conformations is given by the sum of the number of conformations excluded by the two particles separately $\left(\bar{\Omega}^{1 \mathrm{~b}}\right)$, minus the number of conformations excluded by the two particles simultaneously $\left(\bar{\Omega}^{2 b}\right)$,

$$
\bar{\Omega}\left(\mathbf{r}_{1}, \mathbf{r}_{2}\right)=\bar{\Omega}^{1 \mathrm{~b}}\left(\mathbf{r}_{1}\right)+\bar{\Omega}^{1 \mathrm{~b}}\left(\mathbf{r}_{2}\right)-\bar{\Omega}^{2 \mathrm{~b}}\left(\mathbf{r}_{12}\right) \text {. }
$$

Here $\mathbf{r}_{i}(i=1,2)$ denotes the coordinates of particle $i$, and $\mathbf{r}_{i j}=\mathbf{r}_{j}-\mathbf{r}_{i}$. The two-body term $\bar{\Omega}^{2 \mathrm{~b}}\left(\mathbf{r}_{12}\right)$ increases with decreasing distance, and provides an attractive contribution to the polymer-induced interaction $z \bar{\Omega}$. For three colloidal particles the total number of excluded conformations is given by the difference of the one- and two-body contributions $\bar{\Omega}^{1 b}$ and $\bar{\Omega}^{2 b}$, respectively, plus the number of conformations excluded by the three colloidal particles simultaneously $\left(\bar{\Omega}^{3 b}\right)$,

$$
\begin{aligned}
\bar{\Omega}\left(\mathbf{r}_{1}, \mathbf{r}_{2}, \mathbf{r}_{3}\right)= & +\bar{\Omega}^{1 \mathrm{~b}}\left(\mathbf{r}_{1}\right)+\bar{\Omega}^{1 \mathrm{~b}}\left(\mathbf{r}_{2}\right)+\bar{\Omega}^{1 \mathrm{~b}}\left(\mathbf{r}_{3}\right)-\bar{\Omega}^{2 \mathrm{~b}}\left(\mathbf{r}_{12}\right) \\
& -\bar{\Omega}^{2 \mathrm{~b}}\left(\mathbf{r}_{13}\right)-\bar{\Omega}^{2 \mathrm{~b}}\left(\mathbf{r}_{23}\right)+\bar{\Omega}^{3 \mathrm{~b}}\left(\mathbf{r}_{12}, \mathbf{r}_{23}\right)
\end{aligned}
$$

The three-body term $\bar{\Omega}^{3 \mathrm{~b}}\left(\mathbf{r}_{123}\right)$ will increase with decreasing distances. Hence, the three-body contribution provides a repulsive contribution to the polymer-induced interaction $z \bar{\Omega}$. In general, for a system with $N$ colloidal particles, we can write $\bar{\Omega}$ as the sum of one-, two-, three-, and more-body contributions as follows:

$$
\begin{aligned}
\bar{\Omega}\left(\mathbf{r}^{N}\right)= & \sum_{i=1}^{N} \bar{\Omega}^{1 \mathrm{~b}}\left(\mathbf{r}_{i}\right)-\sum_{\substack{\text { pairs } \\
i<j}}^{N} \bar{\Omega}^{2 \mathrm{~b}}\left(\mathbf{r}_{i j}\right) \\
& +\sum_{\substack{\text { triplets } \\
i<j<k}}^{N} \bar{\Omega}^{3 \mathrm{~b}}\left(\mathbf{r}_{i j}, \mathbf{r}_{i k}, \mathbf{r}_{k j}\right)+\cdots .
\end{aligned}
$$

The four- and more-body contributions will give alternating negative (attractive) and positive (repulsive) contributions. Note that the one-body term $\bar{\Omega}^{1 \mathrm{~b}}$ does not depend on the positions of the colloidal spheres, and is therefore constant, except for small variations due to the discreteness of the lattice.

If we were to neglect in the expansion of $\bar{\Omega}[\mathrm{Eq}$. (5)] the three- and more-body terms, we obtain the two-body approximation to the polymer-induced interaction. In this approximation, the effective interaction potential can be written as

$$
U_{\mathrm{col}+\text { pol }}^{2 \mathrm{~b}}\left(\mathbf{r}^{N}\right)=\sum_{\substack{\text { pairs } \\ i<j}}^{N}\left[U_{\mathrm{hs}}\left(\mathbf{r}_{i j}\right)+z \bar{\Omega}^{2 \mathrm{~b}}\left(\mathbf{r}_{i j}\right)\right],
$$

where we ignored the one-body term $\bar{\Omega}^{1 \mathrm{~b}}$, as it does not depend on the colloidal coordinates. For relatively short polymers $U_{\mathrm{col}+\text { pol }}^{2 \mathrm{~b}}$ is expected to be a good approximation, as there will be no, or only few conformations intersecting with three or more colloidal spheres. However, for longer polymers this does not hold, and the two-body approximation is expected to fail.

The thermodynamic potential $F$ corresponding to the partition function $\Xi[\mathrm{Eq}$. (1)] is defined as

$$
F(N, z, V)=-\ln \Xi(N, z, V) \text {. }
$$

We refer to $F$ as the free energy. The pressure is defined as

$$
P=-\left(\frac{\partial F}{\partial V}\right)_{N, z} .
$$

Direct evaluation of this expression using Eqs. (1) and (7) yields the virial expression for the pressure. It contains the derivative of the polymer-induced interaction $z \bar{\Omega}$ with respect to the colloidal coordinates. However, from a computational point of view, Eq. (8) is not a convenient expression to use, as it cannot be reduced into a simple form, due to the many-body character of $\bar{\Omega}$. Therefore, we consider an alternative expression for the pressure, using thermodynamic integration. To that end we write the free energy as 


$$
F(N, z, V)=F(N, z=0, V)+\int_{0}^{z} d z\left(\frac{\partial F}{\partial z}\right)_{N, V} .
$$

Using Eqs. (1) and (7) the integrand can be expressed as an $(N, z, V)$-ensemble average,

$$
\begin{aligned}
\left(\frac{\partial F}{\partial z}\right)_{N, V} & =-\frac{\int_{V} d \mathbf{r}^{N} \Omega\left(\mathbf{r}^{N} ; V\right) \exp \left[-U_{\mathrm{hs}}\left(\mathbf{r}^{N}\right)+z \Omega\left(\mathbf{r}^{N} ; V\right)\right]}{\int_{V} d \mathbf{r}^{N} \exp \left[-U_{\mathrm{hs}}\left(\mathbf{r}^{N}\right)+z \Omega\left(\mathbf{r}^{N} ; V\right)\right]} \\
& =-\langle\Omega\rangle_{N, z, V} .
\end{aligned}
$$

Inserting this expression in $\mathrm{Eq}$. (9) and noting that $F(N, z$ $=0, V)$ is the free energy of a hard-sphere system, we arrive at the following expression for the free energy $F$ :

$$
F(N, z, V)=F_{\mathrm{hs}}(N, V)-\int_{0}^{z} d z\langle\Omega\rangle_{N, z, V} .
$$

Here $F_{\text {hs }}$ denotes the hard-sphere free energy. Inserting this expression for the free energy in the definition of the pressure [Eq. (8)] we obtain

$$
\begin{aligned}
P(\rho, z) & =-\frac{\partial}{\partial V}\left[F_{\mathrm{hs}}(N, V)-\int_{0}^{z} d z\langle\Omega\rangle_{N, z, V}\right] \\
& =P_{\mathrm{hs}}(\rho)+\int_{0}^{z} d z \frac{\partial\langle\Omega\rangle_{N, z, V}}{\partial V},
\end{aligned}
$$

where $P_{\mathrm{hs}}$ denotes the pressure of the hard-sphere system.

The chemical potential $\mu_{\text {col }}$ of the colloidal spheres is defined as

$$
\mu_{\text {col }}=\frac{F+P V}{N} \text {. }
$$

Inserting the relations for the free energy [Eq. (11)] and the pressure [Eq. (12)] yields the following expression for the chemical potential of the colloidal spheres:

$$
\begin{aligned}
\mu_{\mathrm{col}}(\rho, z)= & \mu_{\mathrm{hs}}(\rho)-\frac{1}{N} \int_{0}^{z} d z\langle\Omega\rangle_{N, z, V} \\
& +\frac{1}{\rho} \int_{0}^{z} d z \frac{\partial\langle\Omega\rangle_{N, z, V}}{\partial V},
\end{aligned}
$$

where $\mu_{\mathrm{hs}}$ denotes the chemical potential of the hard-sphere system.

Applying Eqs. (11), (12), and (14) in order to obtain the pressure and colloid chemical potential, requires the knowledge of the hard-sphere equation-of-state and the ensemble average $\langle\Omega\rangle$ of the single-polymer partition function. The Carnahan-Starling expression ${ }^{28}$ yields an accurate expression for the equation-of-state of the hard-sphere fluid. For the hard-sphere solid the free energy is known from simulation ${ }^{29}$ and an accurate analytical expression for the pressure as function of the density has been obtained ${ }^{30}$ by fitting to simulation results. ${ }^{31}$ These will be used in the present work. The dependence of $\langle\Omega\rangle$ on the colloid density and the polymer fugacity has to be determined numerically.

Finally we give the relevant expressions of thermodynamic perturbation theory and scaled-particle theory, applied to the lattice-polymer model and the AO-model. The validity of these theories will be tested against the simulations results. Thermodynamic perturbation theory ${ }^{32}$ provides an approximate expression for the free energy $F$, by writing $F$ as an Taylor-expansion around $z=0$,

$$
F(N, z, V)=F_{\mathrm{hs}}(N, V)-z\langle\Omega\rangle_{z=0}+O\left(z^{2}\right) .
$$

This expansion expresses the free energy of the latticepolymer (or AO) model in terms of the free volume in the $(z=0)$ hard-sphere reference system.

Scaled-particle theory provides an expression for the average volume accessible to a sphere of arbitrary diameter $D$ in a hard-sphere fluid. ${ }^{33,34}$ It is based on an approximate expression for work needed to create a cavity of the size of the a sphere with diameter $D$. When applied to the AO-model to compute the average free volume of a polymer sphere in the $(z=0)$ hard-sphere reference system, ${ }^{26,27}$ it yields the following expression:

$$
\langle\Omega\rangle_{z=0} / V=(1-\eta) \exp \left(-a_{1} \gamma-a_{2} \gamma^{2}-a_{3} \gamma^{3}\right),
$$

with $\eta$ the packing fraction of the colloidal particles $\left(\eta=\rho \sigma^{3} / \sqrt{2}\right), \quad \tilde{\sigma}_{\mathrm{pol}}$ the polymer-colloid size ratio $\left(\tilde{\sigma}_{\mathrm{pol}}=\sigma_{\mathrm{pol}} / \sigma_{\mathrm{col}}\right)$, and

$$
\begin{aligned}
& \gamma=\eta /(1-\eta), \quad a_{1}=3 \tilde{\sigma}_{\mathrm{pol}}+3 \tilde{\sigma}_{\mathrm{pol}}^{2}+\tilde{\sigma}_{\mathrm{pol}}^{3}, \\
& a_{2}=9 \tilde{\sigma}_{\mathrm{pol}}^{2} / 2+3 \tilde{\sigma}_{\mathrm{pol}}^{3}, \quad a_{3}=3 \tilde{\sigma}_{\mathrm{pol}}^{3} .
\end{aligned}
$$

\section{NUMERICAL TECHNIQUES}

\section{A. Computation of polymer partition function}

In the lattice-polymer model for a colloid-polymer mixture, the one-polymer partition function $\Omega\left(r^{N}\right)$ is given by the sum of all possible polymer conformations on a cubic lattice for a given colloid configuration $\mathbf{r}^{N}$. We compute this quantity using the efficient numerical procedure proposed in Ref. 35.

In the lattice-polymer model the presence of the colloidal hard spheres can be described by an external potential that is infinite at the lattice sites occupied by the colloidal spheres, and zero elsewhere. Here we formulate the method to compute the one-polymer partition function $\Omega$ for a general external potential. In addition we show how to compute the average polymer-segment density. For the AO-model, the one-polymer partition function is simply the total number of lattice sites accessible to the polymer spheres.

Consider a linear lattice polymer of length $l$ in an arbitrary external potential. The partition function $\Omega_{l}$ for this polymer, is given by the sum of the Boltzmann weights of all its conformations. (For the sake of clarity, $\Omega$ is labeled with a subscript $l$.) Assume that there is an arbitrary external potential that takes values $U(i)$ at each lattice site $i$. The Boltzmann weight associated with the presence of a single polymer segment on site $i$ is given by $w(i)=\exp [-\beta U(i)]$, with $\beta=1 / k_{B} T$. For the lattice-polymer model we have $w(i)=0$ $[w(i)=1]$ for sites occupied (not occupied) by a colloidal sphere. The Boltzmann weight corresponding with a polymer conformation $\mathbf{r}_{l}$ is given by the product of the Boltzmann weights of the individual segments 


$$
w_{\mathbf{r}_{l}}=\prod_{k=0}^{l} w\left[\mathbf{r}_{l}(k)\right]
$$

where $\mathbf{r}_{l}(k)$ denotes the lattice coordinate of the $k$ th segment of a polymer conformation $\mathbf{r}_{l}$. Hence, the partition function $\Omega_{l}[U]$ can be written as

$$
\Omega_{l}[U]=\sum_{\mathbf{r}_{l}} w_{\mathbf{r}_{l}}=\sum_{\mathbf{r}_{l}} \prod_{k=0}^{l} w\left[\mathbf{r}_{l}(k)\right] .
$$

To compute $\Omega_{l}$ explicitly, we apply a scheme that takes into account all possible polymer conformations. ${ }^{35}$ This is done by generating, for each lattice site $i$, all conformations with length $l$ starting at that site. Simultaneously, the sum of the Boltzmann weights of all generated conformations is computed. The algorithm is recursive. Given all conformations with length $k$ and the corresponding partition function $\Omega_{k}$, the conformations of length $k+1$ are generated and the partition function $\Omega_{k+1}$ is computed. Figure 1 shows a schematic illustration of the method for a three-segment polymer.

Let us first consider a one-segment polymer. All its conformations are generated by placing one segment on each lattice site. The corresponding partition function of the onesegment chain $\Omega_{0}[U]$ is given by the sum of single-segment Boltzmann weights over the lattice,

$$
\Omega_{0}[U]=\sum_{i} w(i)
$$

Next we consider a two-segment polymer. All its conformations can be generated by adding one segment to the conformations of the one-segment polymer. The weight of one particular two-segment conformation with second segment at site $i$ and the first segment at a neighboring site $i^{\prime}$, is given by the product of the weight of the second segment $w(i)$ and the weight of the first segment $w\left(i^{\prime}\right)$. Similarly, the sum of Boltzmann weights of all conformations with second segment at site $i$, denoted by $w_{1}(i)$, is given by the product of $w(i)$ and the sum of the weights $w\left(i^{\prime}\right)$ of all neighboring sites $i^{\prime}$,

$$
w_{1}(i)=w(i) \sum_{i^{\prime}} w\left(i^{\prime}\right) \quad\left(i^{\prime} \in \text { neighbors of } i\right)
$$

This scheme can be continued as follows. We denote the sum of the Boltzmann weights of all conformations of a $k$-segment chain with the $k$ th segment at site $i$ by $w_{k-1}(i)$. Then, the sum of Boltzmann weights of all conformations of a $(k+1)$-segment chain with the $(k+1)$ th segment at site $i$, denoted by $w_{k}(i)$, is given by

$$
w_{k}(i)=n^{\prime}(i) \sum_{i^{\prime}} w_{k-1}\left(i^{\prime}\right) \quad\left(i^{\prime} \in \text { neighbors of } i\right) .
$$

Finally, after applying Eq. (21) $l$ times, we obtain $w_{l}(i)$; the sum of Boltzmann weights of all conformations of ( $l$ $+1)$-segment polymers with the $(l+1)$ th segment at site $i$. Next we can compute the partition function of the $(l+1)$ segment polymer as the sum of the Boltzmann weights $w_{l}$ over all lattice sites,
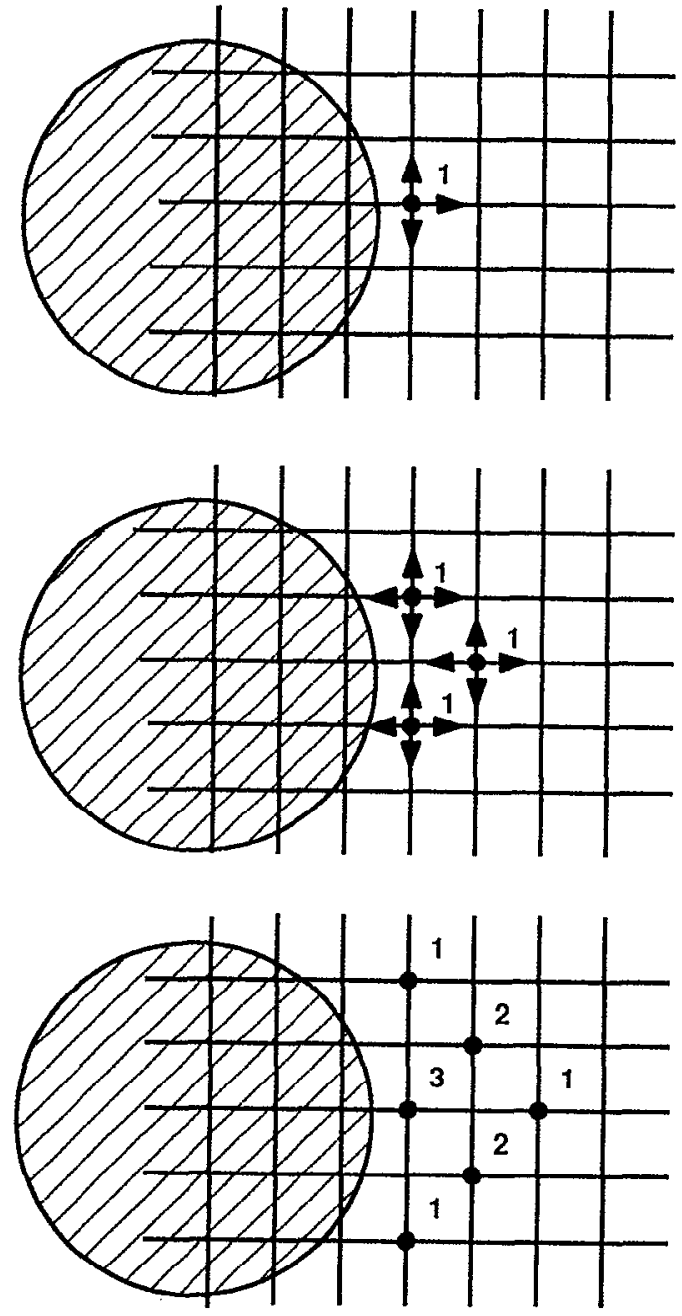

FIG. 1. Iterative scheme [Eq. (21)] to compute the polymer partition function $\Omega$, applied to a three-segment lattice polymer. For clarity we have only shown the method for polymers starting at one particular site. In the full method, polymers start from all possible sites. The large circle represents a colloidal particle. The sites occupied by the colloidal particle are not accessible to the polymers $[w(i)=0]$. The other sites are accessible with equal probability $[w(i)=1]$. The first figure shows the Boltzmann weight of a one-segment polymer at a particular site $i$. The second figure shows the sum of the Boltzmann-weights $w_{1}\left(i^{\prime}\right)$ of all two-segment polymer conformations with the first segment at site $i$. These are obtained by adding (propagating) the one-segment weight of site $i$ to its neighboring sites $i^{\prime}$ and multiply with the single-segment weight $w\left(i^{\prime}\right)$. The third figure shows the sum of the Boltzmann-weights $w_{2}\left(i^{\prime \prime}\right)$ of all three-segment polymers conformations with the first segment at site $i$. These are obtained by adding the twosegment weights of the sites $i^{\prime}$ to their neighboring sites $i^{\prime \prime}$ and multiply with the single-segment weight $w\left(i^{\prime \prime}\right)$.

$$
\Omega_{[}[U]=\frac{1}{2} \sum_{i} w_{l}(i)
$$

The factor $1 / 2$ corrects for the fact that we have counted the contribution of every conformation twice, as we distinguished between the first and the last segment of the chain.

Next we show how to compute the average local segment-density distribution at a site $i$, denoted by $\rho(i)$. According to statistical mechanics, $\rho(i)$ is proportional to the 
sum of the Boltzmann weights of all conformations having a segment at site $i$. We denote this sum by $n(i)$. It can be split up as the sum of Boltzmann weights $n_{0}(i), n_{1}(i), \ldots, n_{l}(i)$ of all conformations having their first, second,..., $(l+1)$ th segment, respectively, at site $i$,

$$
n(i)=\frac{1}{2} \sum_{k=0}^{l} n_{k}(i) .
$$

Here, the factor $1 / 2$ corrects for double counting of the conformations. Clearly, the sum of Boltzmann weights of polymer conformations with their $k$ th segment at site $i$ is equal to the product of the sum of weights of all conformations of polymers with length $k$ ending at site $i$, and those of polymers with length $l-k$ ending at site $i$,

$$
n_{k}(i)=\frac{1}{w(i)} w_{k}(i) w_{l-k}(i),
$$

where the factor $1 / w(i)$ ensures that the single-segment weight $w(i)$ contributes once for each conformation. Combining Eqs. (23) and (24) yields for $n(i)$,

$$
n(i)=\frac{1}{2} \sum_{k=0}^{l} \frac{1}{w(i)} w_{k}(i) w_{l-k}(i) .
$$

Clearly, from computational point of view the iterative scheme [Eq. (21)] is highly attractive. The Boltzmann weights $w(i)$ can be arranged in arrays, the indices of which correspond with the lattice sites $i$. The memory requirements are three real numbers per lattice site; one number corresponds to the old Boltzmann weight $\left[w_{k-1}\right.$ in Eq. (21)], one to the single-segment Boltzmann weight $(w)$, and one to the new Boltzmann weight $\left(w_{k}\right)$. The algorithm corresponding to Eq. (21) is well suited for vector- or parallel processing. In the applications of the scheme to the lattice-polymer model, that will be discussed in the next sections, we have used cubic lattices with sizes in the range $(30)^{3}-(100)^{3}$. These lattices require storage space of 1-25 Mbytes. Typical cputime requirements for these lattice sizes, were $0.001-0.03 \mathrm{~s}$ per propagation step, on a CRAY-YMP supercomputer.

\section{B. Monte Carlo scheme}

Here, we discuss the Monte Carlo technique used to simulate the lattice-polymer model and the AO-model considered in the present work. Both are equivalent to a onecomponent system with an interaction potential of the form given by Eq. (2). For such a system the Metropolis Monte Carlo scheme can be applied straightforwardly. In the Metropolis scheme each subsequent configuration of the colloidal spheres is generated by an attempted move of one (or more) colloidal particle(s). To determine the acceptance probability for this trial move, the change of the interaction potential $U_{\text {col }+ \text { pol }}\left(=U_{\mathrm{hs}}-z \Omega\right)$ has to be computed. The evaluation of the change of the hard-sphere potential $U_{\mathrm{hs}}$ yields a simple check of overlap, and requires a small amount of cpu-time. The change of the polymer-induced interaction $z \Omega$ requires the evaluation of $\Omega$ before and after the trial move. Even when using the efficient propagation method described above, this calculation requires a relatively large amount of cpu-time. In fact, if we would have to compute this difference for every trial move, the requirement would be prohibitive.

To reduce the amount of cpu-time we used a staged Monte Carlo scheme. During the first stage, we generate a sequence of $n$ configurations by $n$ attempted moves of a colloidal sphere, using the Metropolis Monte Carlo scheme. But, rather than using the full (many-body) interaction $U_{\text {col }+ \text { pol }}$, we use the approximate two-body polymer-induced interaction $U_{\mathrm{col}+\text { pol }}^{2 \mathrm{~b}}$ given by Eq. (6). Subsequently, as a second stage, we have to correct for the fact that we did not use the full interaction potential $U_{\mathrm{col}+\mathrm{pol}}$, when generating the sequence of $n$ configurations. We denote the configurations before and after the $n$ "two-body" Monte Carlo moves by $\mathbf{r}^{N}$ and $\mathbf{r}^{N^{\prime}}$, respectively. The correction is achieved by applying the Metropolis Monte Carlo scheme to the configurations $\mathbf{r}^{N}$ and $\mathbf{r}^{N^{\prime}}$ with acceptance probability determined by the change in the difference of the full- and the two-body interaction potential,

$$
\begin{aligned}
& {\left[U_{\mathrm{col}+\mathrm{pol}}\left(\mathbf{r}^{N^{\prime}}\right)-U_{\mathrm{col}+\mathrm{pol}}^{2 \mathrm{~b}}\left(\mathbf{r}^{N^{\prime}}\right)\right]} \\
& \quad-\left[U_{\mathrm{col}+\mathrm{pol}}\left(\mathbf{r}^{N}\right)-U_{\mathrm{col}+\mathrm{pol}}^{2 \mathrm{~b}}\left(\mathbf{r}^{N}\right)\right] .
\end{aligned}
$$

This ensures that the configuration $\mathbf{r}^{N}$ is generated from $\mathbf{r}^{\prime N}$ according to the Metropolis scheme for the interaction potential $U_{\mathrm{col}+\text { pol }}$. Note that if the second-stage move is rejected, the entire sequence of the $n$ "two-body" moves of the first stage is rejected.

In order to apply the staged Monte Carlo scheme we computed prior to a simulation the polymer-induced twobody term $\bar{\Omega}^{2 \mathrm{~b}}$, which was used in tabulated form. The cputime needed to compute the two-body interaction $U_{\mathrm{col}+\text { pol }}^{2 \mathrm{~b}}$ is negligible compared to the time needed for the computation of $U_{\text {col }+ \text { pol }}$. Hence, the use of the staged Monte Carlo scheme reduces the required cpu-time per move with a factor $1 / n$. This would suggest that it is advantageous choose $n$ very large. However, the acceptance of the move from $\mathbf{r}^{N}$ to $\mathbf{r}^{N^{\prime}}$ decreases rapidly with increasing $n$. Hence, for efficient sampling we have chosen $n$ such that a significant fraction of the moves from $\mathbf{r}^{N}$ to $\mathbf{r}^{N^{\prime}}$ is accepted. For the simulations reported here, $n$ was typically of the order of the number of colloidal particles.

\section{SIMULATION RESULTS: (1) POLYMER-INDUCED INTERACTIONS}

The number of excluded conformations in the presence of one, two, and three colloidal hard spheres constitute the first three terms in the expansion Eq. (5) of $\bar{\Omega}$. In the following we use normalized values for the number of excluded conformations, defined by:

$$
\bar{\Omega}^{*} \equiv \frac{\bar{\Omega}}{6^{l} / 2} .
$$

This normalization reduces $\bar{\Omega}$ to an effective excluded volume, expressed in units of the volume of a unit cell of the underlying lattice. In the remainder of this paper we refer to $\Omega$ and $\bar{\Omega}$ also as the free and excluded volume, respectively. 


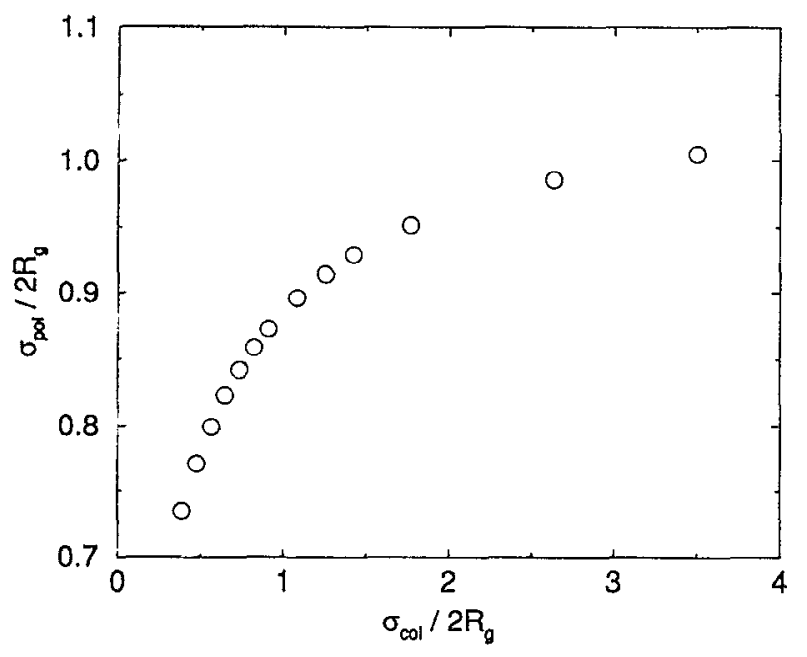

FIG. 2. Mapping between the lattice-polymer model and the AO model. The figure shows, for polymers of length $l=200\left(2 R_{g}=11.8\right)$ and various colloid diameters $\sigma_{\text {col }}$, the polymer-sphere diameter $\sigma_{\mathrm{pol}}$ in the corresponding $\mathrm{AO}$-model, obtained by applying Eq. (28).

\section{A. One-body contribution and relation to the Asakura-Oosawa model}

The one-body contribution $\bar{\Omega}^{1 \mathrm{~b}}$ does not contribute to the polymer-induced interaction $z \bar{\Omega}$ [Eq. (4)], as it is constant, except for small variations due to the discreteness of the lattice. It is, however, interesting to compare the one-body contributions of the lattice-polymer model and the AOmodel, because this provides a means to map the latter model on the former. In the AO-model the volume excluded by one colloidal hard sphere $\bar{\Omega}_{\mathrm{AO}}^{\mathrm{b}}$, is given by

$$
\bar{\Omega}_{\mathrm{AO}}^{\mathrm{lb}}=\frac{\pi}{6}\left(\sigma_{\mathrm{col}}+\sigma_{\mathrm{pol}}\right)^{3}
$$

where $\sigma_{\mathrm{pol}}$ denotes the diameter of the ideal sphere representing a polymer in the AO-model. In order to compare the lattice-polymer model with the AO-model, we define a mapping $\left(l \leftrightarrow \sigma_{\text {pol }}\right)$ by identifying the one-body contributions of the two models,

$$
\bar{\Omega}^{\mathrm{Ib} *}\left(\sigma_{\mathrm{col}}, l\right)=\bar{\Omega}_{\mathrm{AO}}^{\mathrm{Ib}}\left(\sigma_{\mathrm{col}}, \sigma_{\mathrm{pol}}\right) \text {. }
$$

We have computed the one-body contribution $\bar{\Omega}^{1 \mathrm{~b} *}$ for a lattice polymer with length $l=200\left(2 R_{g} \sim 11.8\right)$ and colloidal spheres with diameters $\sigma_{\text {col }}$ in the range 4.5-50. Here $R_{g}$ denotes the radius-of-gyration, which is the typical size of a free lattice polymer. We estimated the radius-of-gyration using the expression, ${ }^{36}$

$$
R_{g}=\sqrt{\frac{l}{6}}
$$

In Fig. 2 we plot the polymer sphere diameter $\sigma_{\mathrm{pol}} / 2 R_{g}$ as a function of the colloid-sphere diameter $\sigma_{\mathrm{col}} / 2 R_{g}$. The figure shows that upon increasing the size of the colloidal sphere, the effective polymer diameter $\sigma_{\mathrm{pol}}$ in the corresponding AOmodel increases considerably. This should be attributed to the fact that a larger colloidal sphere can penetrate less deep
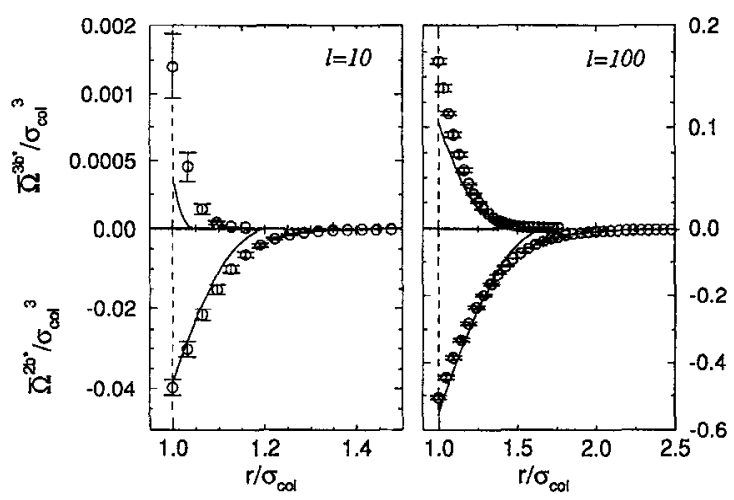

FIG. 3. Simulation results for the two- and three-body contribution to the excluded volume $\vec{\Omega}^{*}$ as function of the distance $r$ between the colloidal spheres $\left(\sigma_{\mathrm{col}}=10.5\right)$. The two figures show the results for "short" $(l=10)$ and "long" ( $l=100)$ polymers. The lower part of each figure gives the results for the normalized two-body contribution. The upper part shows the results for the normalized three-body contributions, where the three colloidal spheres were configurated with their centres on an equilateral triangle. The circles indicate simulation results for the lattice-polymer model, and the error-bars show the mean-square variation in the computed values, due to the discreteness of the lattice. The drawn lines indicate the results for the corresponding AO-models, with polymer-sphere diameters $\sigma_{\text {pol }}=2.18$, and $\sigma_{\text {pol }}=5.2075$, that were computed using Eq. (28).

into the polymer coil, leading to a larger excluded volume $\Omega^{1 b^{*}}$. This, in turn implies a larger polymer sphere diameter $\sigma_{\text {pol }}$.

\section{B. Two- and three-body contributions}

The two- and three-body contribution to the excluded volume $\left(\bar{\Omega}^{2 b}\right.$ and $\left.\bar{\Omega}^{3 b}\right)$ provide an attractive and repulsive contribution to the polymer-induced interaction $z \bar{\Omega}$, respectively. In order to compute these contributions and to determine their dependence on the polymer length, we considered two and three colloidal spheres with diameter $\sigma_{\mathrm{col}}=10.5$ and polymers with lengths $l=10,50,100,200\left(R_{g}=1.55,2.9\right.$, $4.1,5.8)$, and computed $\bar{\Omega}^{2 \mathrm{~b}}$ and $\bar{\Omega}^{3 \mathrm{~b}}$ as function of the mutual positions of the spheres. Here the radius-of-gyration $R_{g}$ is defined by Eq. (29).

The results show that the range of the two- and threebody contributions increases with increasing polymer length. The main feature, however, is that the contribution of the three-body term relative to the two-body term increases strongly with increasing polymer length. To illustrate this we have plotted in Fig. 3 the two- and three-body contributions for "short" $(l=10)$ and "long" $(l=100)$ polymers, as function of the distances between the colloidal particles. Clearly, for "long" $l=100$ polymers the repulsive threebody contribution is a significant fraction of the attractive two-body contribution, whereas for the "short" polymer this contribution is negligible. Hence, for these "long" polymers the polymer-induced interaction has a true many-body character. For the "short" $(l=10)$ polymers the two-body term yields an accurate description of the polymer-induced interaction. Table I lists the results for the coefficients of an exponential fit to the two-body contributions. 
TABLE I. Coefficients of least-squares fits of the normalized two-body contribution $\Omega^{\text {th }} / \sigma_{c}^{*}$ as function of the reduced distance $r^{*}=r / v_{\text {col }}$ [fit function $\left.-\exp \left(a_{1}+a_{1} r^{*}+a_{2} r^{r 2}\right)\right]$, for various polymer lengths $l$ and colloidal spheres with diameter $\sigma_{c e l}=10.5$.

\begin{tabular}{rrrr}
\hline \hline \multicolumn{1}{c}{$l$} & \multicolumn{1}{c}{$a_{0}$} & \multicolumn{1}{c}{$a_{1}$} & \multicolumn{1}{c}{$a_{2}$} \\
\hline 10 & -12.55 & 27.288 & -17.955 \\
50 & -0.381 & 1.998 & -3.015 \\
100 & 1.010 & -0.278 & -1.385 \\
200 & 1.752 & -1.038 & -0.642 \\
\hline \hline
\end{tabular}

For comparison we have also plotted in Fig. 3 the results for the corresponding $A O$-models, with the correspondence defined by $\mathrm{Eq}$. (28). We ohserve a slightly shorter range of the two body contribution and (significant) lower values of the three-body contribution compared to the lattice-polymer model. Both effects should be attributed to the fact that there are a significant number of lattice-polymer conformations with a spatial extent larger than the polymer-sphere in the AO-model.

\section{SIMULATION RESULTS: (2) THERMODYNAMIC PROPERTIES}

The local structure of the colloidal component shows a significant change upon the addition of polymer. ${ }^{25}$ The main feature is the enhancement of the colloidal radial-distribution function at contact, due to the polymer-induced attraction. Figure 4 shows snapshots of the final colloid configuration and the corresponding local polymer segment concentration of a Monte Carlo simulation at relatively high polymer fugacity. The distinct regions with high and low concentrations observed in this snapshot are an indication for the occurrence of a phase separation. In order to investigate the phase behavior more systematically we have performed an extensive series of numerical simulations for various polymer lengths, using the Monte Carlo scheme described in Sec. 1II. We computed the free volume $\langle\Omega\rangle$ as function of the colloid density $\rho_{\text {col }}$ and the polymer fugacity $z$. From these results we obtained the thermodynamic properties, such as the pressure and the chemical potential of the colloidal particles. In turn, these results allowed to determine the phase boundaries. For the sake of comparison, we also performed simulations of the AO-model corresponding to lattice polymer models with "long" polymers.

We considered colloidal hard spheres with diameter $\sigma_{\text {col }}=10.5$ and lattice polymers with length $l=10, l=50$, and $l=100$. In the $\Lambda O_{- \text {models }}\left(\sigma_{c o l}=10.5\right)$ corresponding to the $l=50$ and $l=100$ lattice models, the polymer sphere diameters were $\sigma_{\mathrm{pol}}=5.2075$, and $\sigma_{\mathrm{pol}}=7.3104$, respectively. Typically we performed simulations of $N=108$ (densities in the fluid range), or $N=256$ (densities in the solid range) colloidal hard spheres in a cubic box with periodic boundary conditions. Each simulation run consisted of an initial equilibration stage of $2000-5000$ cycles, and a subsequent production run of 10000-20000 cycles, where one cycle corresponds to one attempted Monte Carlo move per particle.

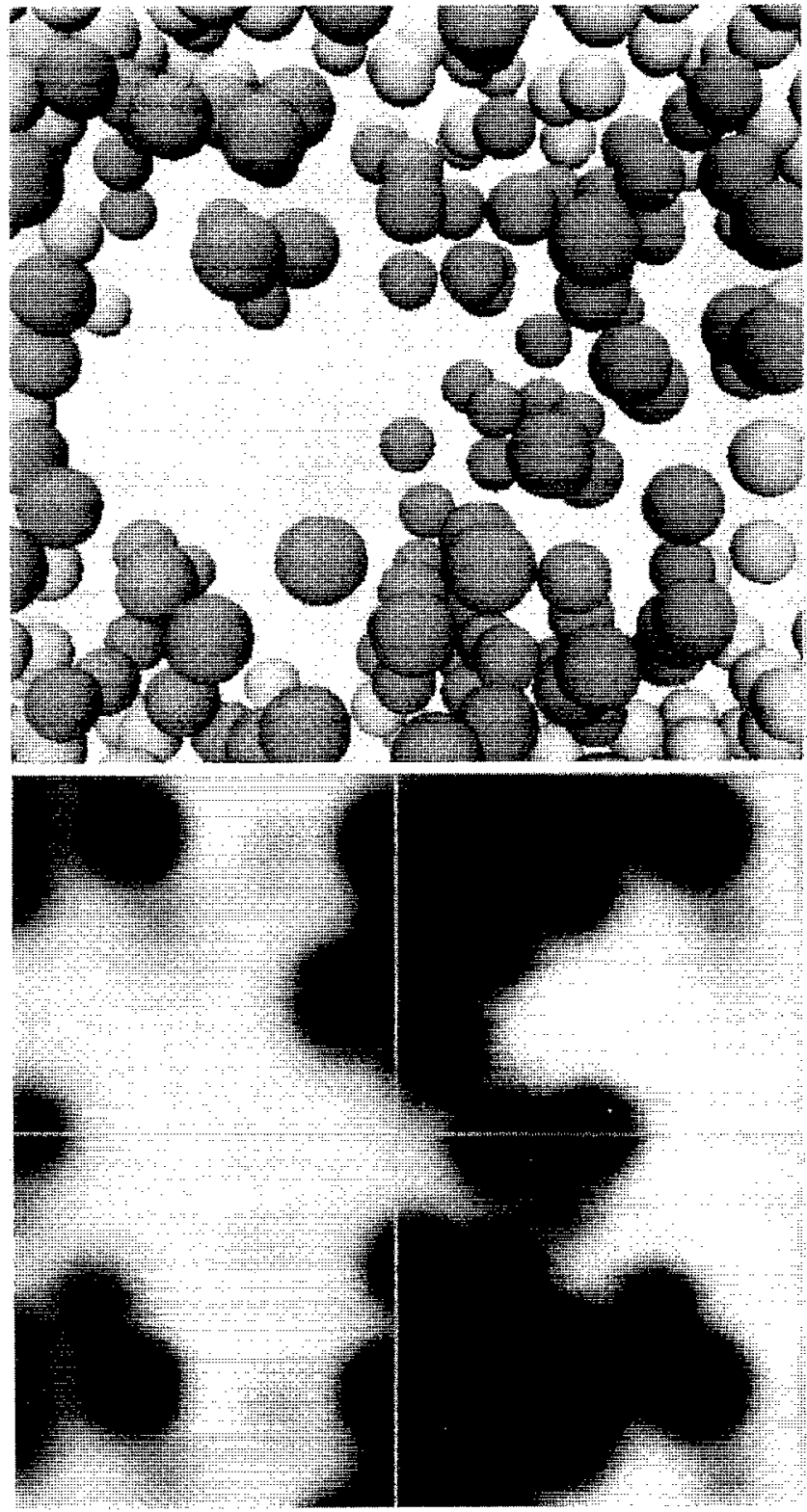

FIG. 4. Snapshot of a simulation of a lattice polymer model for a colloid dispersed in a polymer solution, with colloidal spheres of diameter $\sigma_{\text {tol }}=10.5$ and polymers of lengths $l=50$ at relatively high polymer fugacity $z^{*} \sigma_{\text {col }}^{3}=17\left(z^{*}=z^{*} \times 6^{4} / 2\right)$. The average colloid density is $\rho_{\mathrm{col}} \rho_{\mathrm{col}}^{3}=0.25$. The upper figure shows the colloidal spheres. In the lower figure we have shown the corresponding polymer-segment density in a thin slice cut through the system, with the slice in the plane of the paper. The color-coding is white to black, for high to low polymer-segment density, respectively. The segment concentration is computed using Eq. (25). We observe that there are distinct regions with low and high concentrations. It indicates that the colloid has phase separated into a low and a high density phase, with a high and low polymer-segment concentration, respectively.

\section{A. Free volume}

\section{Lattice-polymer model}

The results for the free volume in the $(z=0)$ hard-sphere reference system in lattice-polymer models with $l=10$ and $l=100$ are shown in Fig. 5 . In this figure we have plotted both the relative free volume $\left\langle\Omega^{*}\right\rangle_{z=0} / V$ (upper part), and 


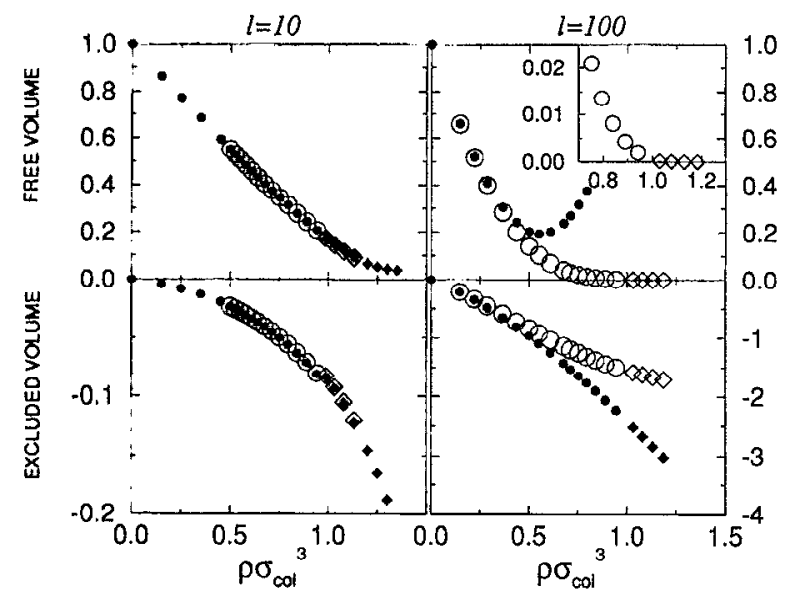

FIG. 5. Average free and excluded volume in the lattice-polymer model $\left(\sigma_{\mathrm{col}}=10.5\right)$ for polymers of length $l=10$ and $l=100$, as function of the colloid density $p$. The figures shows free and excluded volume in the $(z=0)$ hard-sphere reference system obtained by Monte Carlo simulation of $N=108$ (fluid) and $N=256$ (solid) colloidal spheres. The upper part of the figures shows the relative tree volume $\left\langle\Omega^{*}\right\rangle_{z=0} / V$, where the inset $(l$ $=100$ ) is a magnification of the high-colloid density region. The lower part of the figures shows the excluded volume per particle minus the (constant) one-body contribution, $\left\langle\bar{\Omega}^{*}\right\rangle_{z=0} / N-\tilde{\Omega}^{1 b *}$. For both the upper and the lower figures the open symbols indicate the results for the true free and excluded volume, whereas the filled symbols indicate the results for the free and excluded volume without the three- and more-body contributions. The circles and diamonds refer to simulations of the colloidal fluid and solid, respectively.

the excluded volume per particle $\left\langle\bar{\Omega}^{*}\right\rangle_{z=0} / N$ (lower part). We recall that the latter is proportional to the polymerinduced interaction per colloidal sphere. Clearly, for increasing colloid density the relative free volume decreases. In particular for the "long" $(l=100)$ polymers the relative free volume becomes very small at high colloid densities. At these densities these "long" polymers hardly fit between the colloidal spheres.

To quantify the results for the free volume in the $(z=0)$ hard-sphere reference system we have fitted the results. For the densities in the fluid range we have fitted the relative free volume $\left\langle\Omega^{*}\right\rangle_{2=0} / V$ to the scaled-particle expression Eq. (16) for the free volume in the AO-model, with $a_{1}, a_{2}$, and $a_{3}$ as fit parameters. The results of the fits are listed in Table II.

TABLE II. Coefficients of least-squares fits to simulation results for the relative free volume $\left\langle\Omega^{*}\right\rangle_{z=0} / V$ as function of colloid density, for densities in the fluid range $\left(0.0 \leqslant \rho \sigma_{\mathrm{col}}^{3} \leqslant 0.70\right)$. The table lists results for three lattice-polymer models (upper part) and two AO-models (lower part). The fit function is given by the scaled-particle expression for the free volume in the AO-model Eq. (16).

\begin{tabular}{ccrc}
\hline \hline$l$ & $a_{1}$ & \multicolumn{1}{c}{$a_{2}$} & \multicolumn{1}{c}{$a_{3}$} \\
\hline 10 & -0.314 & 0.0361 & 0.0007 \\
50 & -1.539 & -0.0043 & 0.0444 \\
100 & -2.775 & -0.0481 & 0.0748 \\
& & & \\
$\sigma_{\text {pol }}$ & & & \\
5.2075 & -1.561 & -0.153 & 0.027 \\
7.3104 & -2.742 & -0.676 & 0.146 \\
\hline \hline
\end{tabular}

TABLE III. Coefficients of least-squares fits to simulation results for the relative free volume $\left\langle\Omega^{*}\right\rangle_{z=0} / V$ as function of the reduced density $\rho^{*}=\rho \sigma_{\text {col }}^{3}$ for densities in the solid range $\left(0.70 \leqslant \rho \sigma_{\text {col }}^{3} \leqslant 0.85\right)$. The table lists results for three lattice-polymer models (upper part) and the AO-model (lower part). For $l=10$ the fit function was taken to be polynomial, $a_{0}+a_{1} \rho^{*}+a_{2} \rho^{* 2}+a_{3} \rho^{* 3}$. For $l=50$ and $l=100$, and the AO-models the fit function was taken to be exponential, $\exp \left(a_{0}+a_{1} \rho^{*}+a_{2} \rho^{* 2}\right)$. The relative free volume in the AO-model with $\sigma_{\mathrm{pol}}=7.3104$ was less than $10^{-6}$.

\begin{tabular}{crrrc}
\hline \hline$l$ & \multicolumn{1}{c}{$a_{0}$} & \multicolumn{1}{c}{$a_{1}$} & \multicolumn{1}{c}{$a_{2}$} & \multicolumn{1}{c}{$a_{3}$} \\
\hline 10 & 1.4791 & -1.8385 & 0.3826 & 0.140624 \\
50 & -16.2159 & 33.4582 & -22.0591 & $\cdots$ \\
100 & 6.8327 & -4.9792 & -9.7681 & $\cdots$ \\
$\sigma_{\text {pol }}$ & & & & \\
5.2075 & -79.5038 & 158.526 & -85.6174 & $\cdots$ \\
\hline \hline
\end{tabular}

Coefficients of a polynomial fit of the relative free volume for densities in the solid range are listed in Table III.

In order to determine the dependence of the free volume on the polymer fugacity $z$ we performed a series of Monte Carlo simulations at finite values of $z$, for systems with $l=50$ and $l=100$ polymers. The results for the $l=50$ lattice-polymer model are plotted in Fig. 6. The increase of the free volume upon increasing fugacity, at fixed colloid

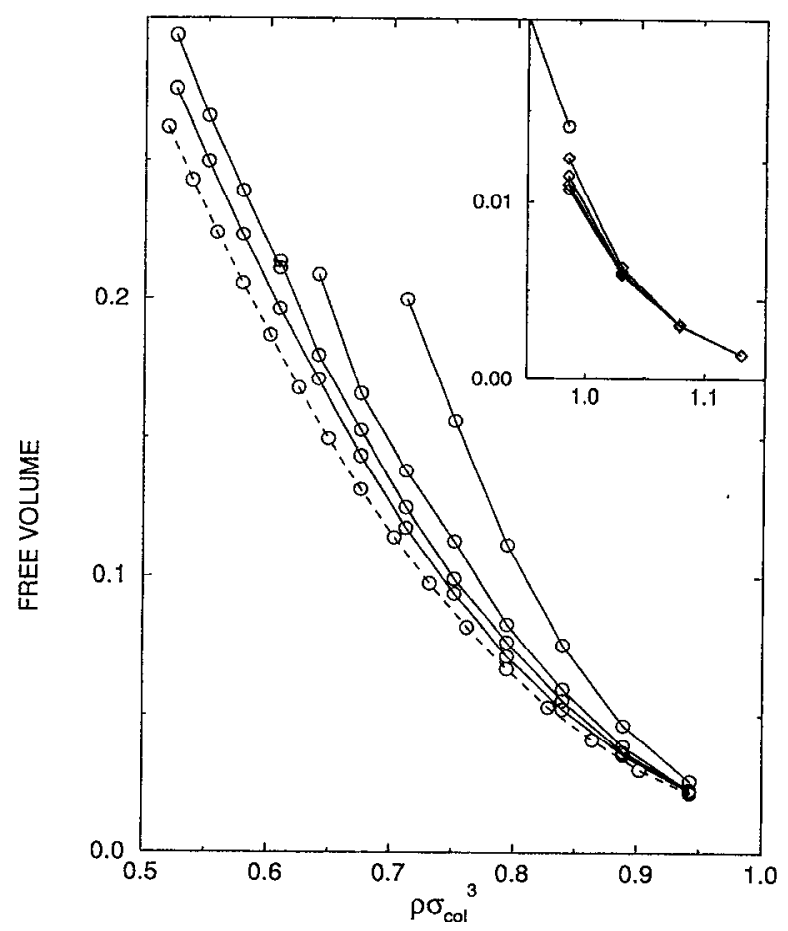

FIG. 6. Average relative free volume $\left\langle\Omega^{*}\right\rangle / V$ in the lattice polymer model $\left(\sigma_{\mathrm{col}}=10.5\right)$ for polymers of length $l=50$ as function of the colloid density $\left(\rho \sigma_{c o l}^{3}\right)$, for various values of the polymer fugacity $z$. The data in the dense fluid range (circles), and solid range (inset; diamonds) are obtained by Monte Carlo simulation of systems with $N=108$ and $N=256$ colloidal spheres, respectively. The lines connect the data points with the same polymer fugacity $z$, where the dashed line corresponds to $z=0.0$. From the bottom to the top the drawn lines correspond successively with $z^{*} \sigma_{\text {col }}^{3}=5.8$, $9.3,11.6$, and 23.2 for the fuid state points, and $z^{*} \sigma_{\mathrm{col}}^{3}=11.6,17.4$, and 23.2 for the solid state points, respectively $\left(z^{*}=z \times 6^{l} / 2\right)$. 


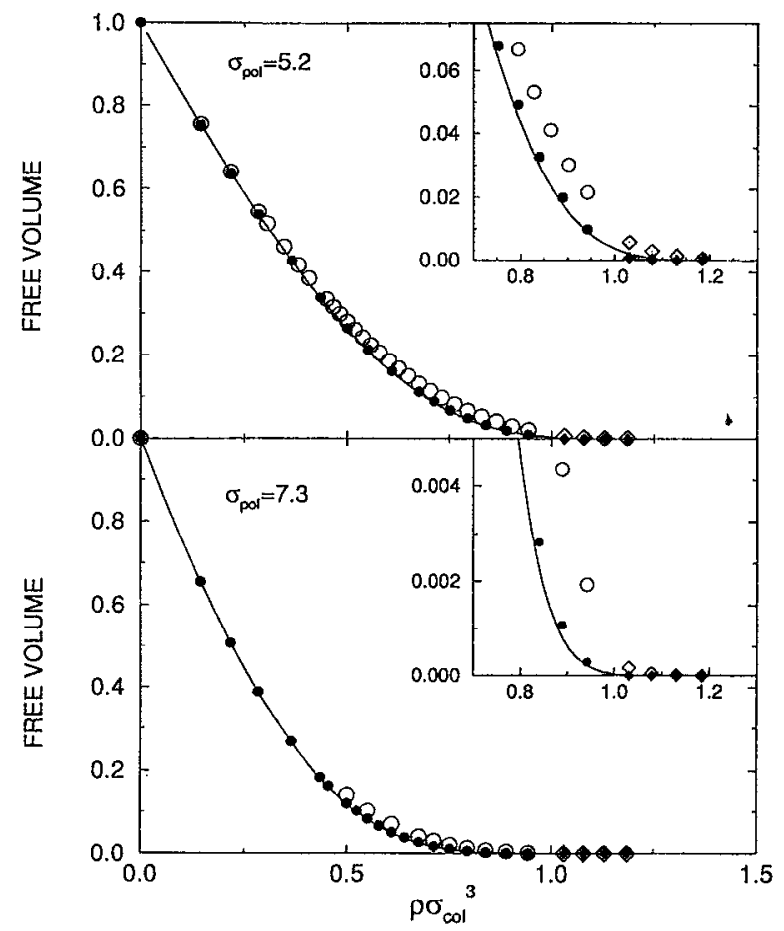

FIG. 7. Average relative free volume $\left(\left\langle\Omega^{*}\right\rangle_{z=0} / V\right)$ in the AO-model $\left(\sigma_{\mathrm{col}}=10.5\right)$ for polymer spheres with diameters $\sigma_{\mathrm{pol}}=5.2075$ and $\sigma_{\mathrm{pol}}=7.3104$, as function of the colloid density $\rho$. These polymer-sphere diameters correspond with lattice-polymers of length $l=50$ and $l=100$, respectively. The figures show the free volume in the $(z=0)$ hard-sphere reference system obtained by Monte Carlo simulation of $N=108$ (fluid) and $N=256$ (solid) colloidal spheres. The inset is a magnification of the region with high colloid densities. The solid symbols show the results for the AOmodel, where the circles and diamonds refer to simulations of a colloidal fluid and solid, respectively. The open symbols and the drawn line are shown for comparison. They indicate the results for the corresponding lattice-polymer model and the prediction of scaled particle theory (Refs. 43 and 26).

density, reflects the enhanced contribution of colloidconfigurations with a high value of the free volume, due to the $-z \Omega$ term in the interaction potential Eq. (2). For the regions were the free volume decreases relatively sharply with increasing density $\left(z^{*} \sigma_{\mathrm{col}}^{3}=23.2\right)$ snapshots of the colloid configurations showed a nonuniform distribution over the simulation box. This suggests that these state points are unstable. Below, we show that this is indeed the case. The $z$-dependence for the $l=100$ lattice-polymer model is qualitatively the same as for the for the $l=50$ model.

\section{Asakura-Oosawa model}

Next we consider the simulation results for the free volume of the AO-model, with $\sigma_{\mathrm{pol}}=5.2075$ and $\sigma_{\mathrm{pol}}=7.3104$. Figure 7 shows the results for the $(z=0)$ hard-sphere reference system, where we have plotted also the results for the corresponding lattice-polymer models. If we compare the two models, we observe lower values for the free volume in the AO-model. The relative difference increases with increasing colloid densities. This should be attributed to the fact that at high densities "nonspherical" conformations of the lattice polymer, that arc absent in the AO-model, have an enhanced contribution to the fraction of accessible conformations. An additional observation is that the relative difference hetween the AO- and lattice polymer model is more pronounced for the longer polymers. Apparently the contribution of the "nonspherical" conformations becomes larger for longer polymers. The contribution of "nonspherical" conformations is extremely enhanced at solid densities, where the AO-model strongly underestimates the number of possible conformations. Results of fits of the free volume in the AO model in the fluid range, with a fit function given by $\mathrm{Eq}$. (16), are listed in Table II. The coefficients of a polynomial fit of the free volume in the solid range are listed in Table III. We have also determined the dependence of the free volume on the polymer fugacity. They turn out to be qualitatively the same as for the $l=50$ lattice-polymer model, shown in Fig. 6.

\section{B. Phase behavior}

At zero polymer-fugacity the lattice-polymer model and the AO-model turn into a hard-sphere system, that exhibits the well-known fluid-solid coexistence at a pressure $P \sigma_{\text {col }}^{3}=11.70$, with densities $\rho_{\mathrm{fl}} \sigma_{\mathrm{col}}^{3}=0.943$ and $\rho_{\mathrm{sol}} \sigma_{\mathrm{col}}^{3}=1.041,{ }^{29}$ for the coexisting fluid and solid phase, respectively. Upon increasing the polymer fugacity, the increase of the polymer-induced attraction will induce a change in the location of the fluid-solid coexistence point. In addition a "vapor-liquid" phase separation might appear. There is experimental (see e.g., Refs. 12-14, 16, 17) and theoretical evidence (see, e.g., Refs. 13,24,26 that a colloidpolymer mixture exhibits either a "vapor-liquid-solid"-like or a "vapor-solid"-like phase diagram.

By simulation we determined the phase diagrams of the lattice-polymer model with "short" $(l=10)$ and with "long" ( $l=50$ and $l=100)$ polymers. The phase boundaries were determined by locating the state points in two different phases with equal pressure and equal chemical potentials of the colloidal and polymer component. For the latticepolymer model, the chemical potential of the polymers $\mu_{\mathrm{pol}}=\ln (z)$ is an imposed parameter, whereas the pressure $P$ and the colloid chemical potential $\mu_{\text {col }}$ have to be computed. For comparison we also determined, by simulation, the phase diagram of the AO-models, corresponding to the latticepolymer model with the "long" $(l=50$ and $l=100)$ polymers.

\section{1. "Short" polymers}

For the "short" polymers $\left(l=10,2 R_{g} / \sigma_{\text {col }} \simeq 0.25\right)$ the polymer-induced interaction $z \bar{\Omega}$ is, to good approximation, pairwise additive in the colloidal coordinates (see Ref. 25 and Sec. IV B). This allows us to study the lattice-polymer model with these "short" polymers by ordinary constantpressure Monte Carlo simulation of a one-component system with an interaction potential given by Eq. (6). For the polymer-induced interaction we used a fit to the simulation results for the two-body contribution $\bar{\Omega}^{2 b}$, listed in Table I.

To compute the phase diagram we used a scheme recently introduced by Kofke, ${ }^{37,38}$ that enables direct simulation of the phase coexistence line, given the coexistence data of an initial point. The method makes use of the Gibbs- 


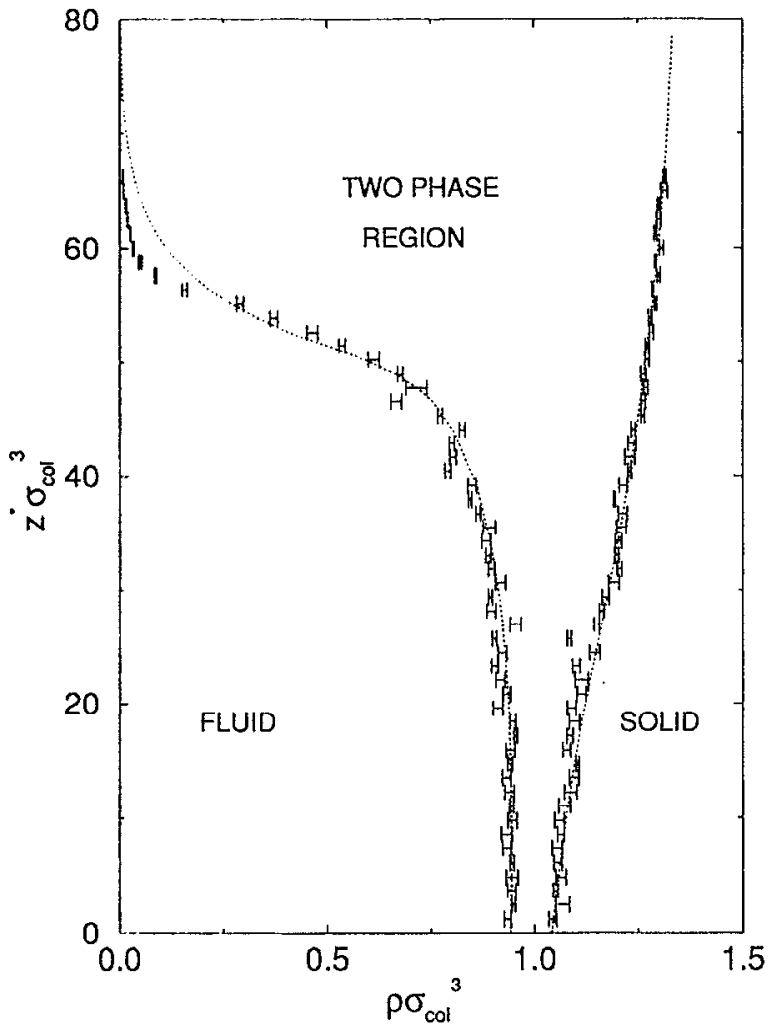

FIG. 8. Phase diagram of lattice-polymer model $\left(\sigma_{\mathrm{col}}=10.5\right)$ for polymers of length $l=10$. The points with error bars indicate the reduced densities $\rho \sigma_{i=1}^{3}$ of the coexisting phases as function of the reduced polymer fugacity $z^{*} \sigma_{\mathrm{col}}^{3}\left(z^{*}=z \times 6^{l} / 2\right)$ obtained by Monte Carlo simulation. The dotted lines indicate the results for the phase boundaries obtained by applying first-order perturbation theory to simulation results for the $(z=0)$ hard-sphere reference system.

Duhem equation applied to coexisting phases. For the lattice-polymer model the Gibbs-Duhem equation yields the following relation between the pressure $P$ and the polymer fugacity $z$ along the $P-z$ coexistence line:

$$
\left(\frac{\partial P}{\partial z}\right)_{\text {coex }}=-\frac{\Delta\langle\bar{\Omega}\rangle}{\Delta V}
$$

where $\Delta\langle\bar{\Omega}\rangle$ and $\Delta V$ denote the difference of $\langle\bar{\Omega}\rangle$ and the volume $V$ of the two coexisting phases. This equation was solved using a 4-points Adams-Bashforth-Moulton predictor-corrector algorithm (see, e.g., Ref. 39), starting from the $(z=0)$ hard-sphere fluid-solid coexistence point. Both after the predictor and after the corrector step the righthand side of Eq. (30) was evaluated by one constant pressure Monte Carlo simulation for each phase. In Fig. 8 we have plotted the resulting phase-diagram in the $\rho-z$ plane. The most important feature is the absence of a liquid phase; there is no liquid-vapor phase separation of the fluid phase for these "short" polymers.

\section{2. "Long" polymers}

For the lattice-polymer models with "long" polymers $\left(l=50,2 R_{g} / \sigma_{\text {col }}=0.55\right.$ and $\left.l=100,2 R_{g} / \sigma_{\text {col }} \simeq 0.75\right)$, the polymer-induced interaction $z \bar{\Omega}$ has a significant three- and

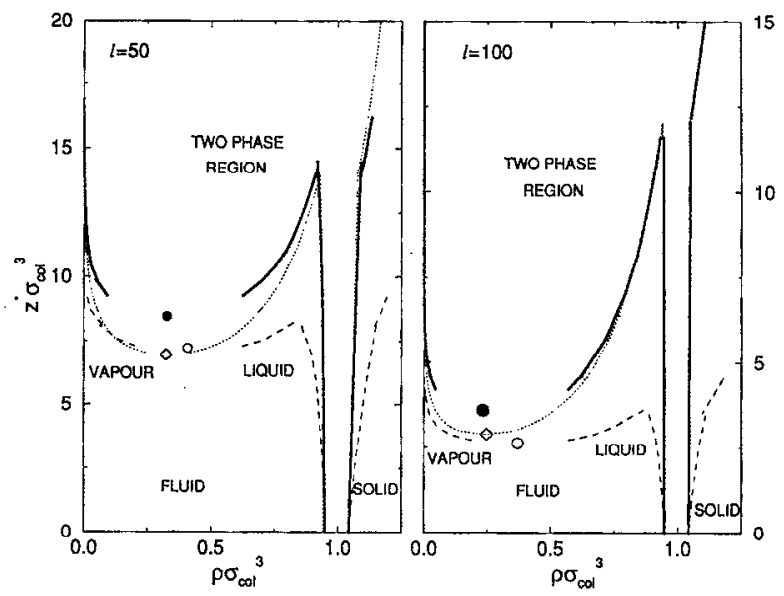

FIG. 9. Phase diagrams of lattice-polymer model $\left(\sigma_{\mathrm{col}}=10.5\right)$ for polymers with lengths $l=50$ and $l=100$. The figures show the reduced densities $\rho \sigma_{\mathrm{col}}^{3}$ of the coexisting phases as function of the reduced polymer fugacity $z^{*} \sigma_{\mathrm{col}}^{3}$ $\left(z^{*}=z \times 6^{l} / 2\right)$. The drawn lines indicate the simulation result taking into account the full polymer-induced interaction (black circle indicates estimated critical point). The dashed lines indicate the simulation result obtained if the three- and more-body terms in the polymer-induced interaction are neglected (two-body approximation). The open circle indicates the estimated critical point in the two-body approximation. The dotted lines indicate the result obtained by applying first-order perturbation theory to simulation results for the $(z=0)$ hard-sphere reference system (diamond indicates estimated critical point).

more-body contribution (see Ref. 25 and Sec. IV B). Consequently, direct application of methods for direct simulation of phases coexistence is not possible. Hence, we determined the phase coexistence by thermodynamic integration.

The pressure $P$ and the colloid chemical potential $\mu_{\mathrm{col}}$ for the high-density fluid states $\left(\rho \sigma_{\mathrm{col}}^{3} \geqslant 0.5\right)$, and the solid states were determined using Eqs. (12) and (14), where we inserted polynomial fits of the free volume $\left\langle\Omega^{*}\right\rangle_{z, \rho}$ in $\rho$ and $z$. For the low-density fluid states we determined for various values of $z^{*}$ the excess colloid chemical potential $\mu_{\mathrm{col}}^{\mathrm{ex}}$ as a function of the colloid density using the Widom particleinsertion method. ${ }^{40}$ This method is based on the following expression for the chemical potential:

$$
\begin{aligned}
\mu_{\mathrm{col}}^{\mathrm{ex}}(\rho, z)= & -\operatorname{In}\left\langle e^{-\left[U_{\mathrm{hs}}\left(\mathrm{r}^{N+1}\right)-U_{\mathrm{hs}}\left(\mathrm{r}^{N}\right)\right]}\right. \\
& \left.\times e^{z\left[\Omega\left(\mathrm{r}^{N+1} ; V\right)-\Omega\left(\mathrm{r}^{N} ; V\right)\right]}\right\rangle_{\rho, z} .
\end{aligned}
$$

The ensemble average on the right-hand side is determined by random insertions of the $(N+1)$-th sphere in a Monte Carlo simulation of a system with $N$ colloidal spheres. The pressure $P$ was computed from the results for the chemical potential using the Gibbs-Duhem relation,

$$
P(\rho, z)=\rho+\int_{0}^{\rho} d \rho^{\prime} \rho^{\prime} \frac{\partial \mu_{\mathrm{col}}^{\mathrm{ex}}}{\partial \rho^{\prime}}\left(\rho^{\prime}, z\right) .
$$

Figure 9 shows the computed phase diagrams for the $l=50$ and $l=100$ lattice-polymer models. The most important observation is the occurrence of a "vapor-liquid"-like phase separation of the colloidal fluid, that was not observed in the phase-diagram of the "short" $(l=10)$ polymers (Fig. 8). Apparently, an increase in the range of the polymer- 
TABLE IV. Estimated values of the fugacity $z$ and density $\rho$ at the critical and triple point for three lattice polymer models (upper part) and two $\mathrm{AO}$ models (lower part) $\left(z^{*}=z \times 6^{l} / 2\right)$. The table lists values obtained by simulation (SIM), and values obtaincd by applying two approximate theories, the two-body approximation (TH-2B) and first-order thermodynamic perturbation theory (TH-PB).

\begin{tabular}{cccccc}
\hline \hline$l$ & & $z_{\text {crit }}^{*} \sigma_{\text {col }}^{3}$ & $\rho_{\text {crit }} \sigma_{\text {col }}^{3}$ & $z_{\text {trip }}^{*} \sigma_{\text {col }}^{3}$ & $\rho_{\text {trip }} \sigma_{\text {col }}^{3}$ \\
\hline 50 & SIM & 8.5 & 0.33 & 14.0 & 0.92 \\
& TH-2B & 7.2 & 0.41 & 8.3 & 0.83 \\
& TH-PB & 6.9 & 0.33 & 13.9 & 0.93 \\
100 & SIM & 3.6 & 0.27 & 11.9 & 0.94 \\
& TH-2B & 2.7 & 0.37 & 3.6 & 0.87 \\
& TH-PB & 2.9 & 0.35 & 11.9 & 0.94 \\
$\sigma_{\text {pol }}$ & & & & & \\
5.2075 & SIM & 9.0 & 0.40 & 12.9 & 0.93 \\
\multirow{2}{*}{7.3014} & TH-PB & 6.9 & 0.36 & 12.9 & 0.93 \\
& SIM & 3.7 & 0.29 & 11.9 & 0.94 \\
& TH-PB & 2.9 & 0.27 & 11.9 & 0.94 \\
\hline \hline
\end{tabular}

induced attraction does lead to the stabilization of a dense disordered phase. We can identify a critical and triple fugacity as the fugacities where the liquid appears and disappears, respectively. These are analogous to the reciprocal critical and triple temperature known from the phase diagrams of simple molecular fluids. Close to the critical point we were not able to determine the phase-coexistence due to large fluctuations. However we could estimate the location of the critical point by extrapolating the results for the vapor-liquid coexistence densities above the critical fugacity using the law of rectilinear diameters ${ }^{41}$ and the scaling law for the liquid-vapor density difference at coexistence. ${ }^{42}$ Clearly, the range of stability of the liquid range, i.e., the ratio of the triple and critical fugacity, increases with increasing polymer length. Table IV lists the estimated locations of the critical points and triple points. If we assume that the ratio of the triple and critical fugacity depends linearly on the polymercolloid size ratio $2 R_{g} / \sigma_{\text {col }}$, we can estimate from the simulation results that the liquid phase disappears for polymercolloid size ratios $2 R_{g} / \sigma_{\text {col }} \leqslant 0.45$.

In Fig. 10 we have plotted the computed phase diagrams of the AO-models. For comparison we have plotted in the same figure the results for the corresponding lattice-polymer models. Clearly, the results for the AO-model compares quite well with that of the lattice-polymer model. Apparently, for the range of polymer fugacities considered here, the significant lower values of the free volume at high colloid densities (see Fig. 7) do have little or no influence on the phase diagram. Table IV lists the estimated locations of the critical point and triple points.

\section{VALIDITY OF THEORETICAL APPROXIMATIONS}

There have been several theoretical studies of the thermodynamic properties of colloid-polymer mixtures. ${ }^{13,14,20,21,24,26,27}$ Most of these studies concerned the AO model or a closely related model. In all theoretical studies, approximations had to be made in order to arrive at quantitative predictions. Here we discuss a comparison of

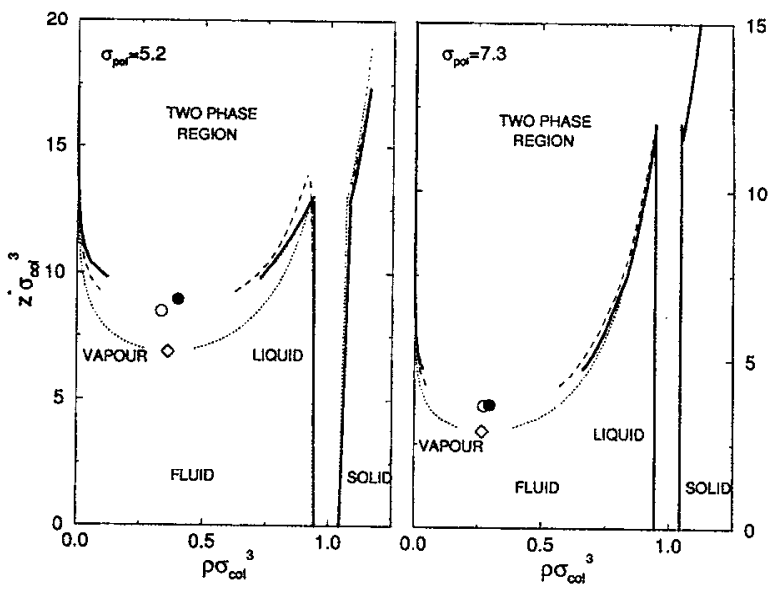

FIG. 10. Phase diagrams of AO model $\left(\sigma_{\mathrm{col}}=10.5\right)$ for polymer-sphere diameters $\sigma_{\mathrm{pol}}=5.2075$ and $\sigma_{\mathrm{pol}}=7.3104$. These polymer-sphere diameters correspond with lattice-polymers of length $l=50$ and $l=100$, respectively. The figures show the reduced densities $\rho \sigma_{\mathrm{col}}^{3}$ of the coexisting phases as function of the reduced polymer fugacity $z^{*} \sigma_{\text {col }}^{3}\left(z^{*}=z \times 6^{l} / 2\right)$. The drawn lines indicate the simulation result taking into account the full polymerinduced interaction (black circle indicates estimated critical point). The dashed lines indicate the simulation result for the corresponding latticepolymer model (open circle indicates estimated critical point). The dotted lines indicate the result obtained by applying first-order perturbation theory to the simulation results for the $(z=0)$ hard-sphere reference system (diamond indicates estimated critical point).

our simulation results presented in Sec. V with results obtained by using the most common approximations.

\section{A. Two-body approximation}

In the two-body approximation [Eq. (6)] the three- and more-body contributions to the polymer-induced interaction are neglected. In order to determine the role of the manybody part on the thermodynamic properties, we computed, by Monte Carlo simulation, the free volume and the phase diagram in the two-body approximation and compared these with the true many-body results.

Figure 5 shows, along with the true many-body results, the two-body results for the excluded (and free) volume for "short" $(l=10)$ and "long" $(l=100)$. Clearly, for the "short" $(l=10)$ polymers the two-body approximation provides an accurate estimate for the excluded volume, consistent with the observation that the three-body contribution is negligible for these "short" polymers (see Fig. 3). However, for the "long" ( $l=100)$ polymers the excluded volume is underestimated significantly in the two-body approximation, in particular at high colloid densities. For these "long" polymers and at these colloid densities, the three-, and more-body terms give a significant positive contribution to the excluded volume, or, equivalently, a significant positive contribution to the polymer-induced interaction. Again, this is consistent with the observation (see Fig. 3) that for these "long" polymers the positive three-body contribution is a significant fraction of the negative two-body contribution. The increasing difference for increasing densities should be attributed to the increasing probability of the occurrence of clusters of three and more colloidal spheres at higher colloid densities.

The two-body results for the phase diagram of the 
"long" $(l=50$ and $l=100)$ polymers are plotted in Fig. 9, along with the true many-body results. Clearly, neglecting the three- and more body contributions leads to a significant shift of the critical polymer fugacity to lower values. This is a direct consequence of the fact that for these "long" polymers, the strength of the polymer-induced attraction is strongly overestimated in the two-body approximation. In addition the two-body approximation predicts a lower value of the ratio of the triple and critical fugacity, that is, partly, due to the enhanced widening of the liquid-solid coexistence region in the two-body result. Apparently, in the two-body approximation the stability of the liquid phase with respect to the solid phase is underestimated. This could be anticipated from the results for the excluded volume, that is proportional to polymer-induced interaction, shown in Fig. 5. The figure shows that for "long" $(l=100)$ polymers, the two-body approximation increasingly underestimates the polymerinduced interaction with increasing density. Hence, in the two-body approximation the states at high densities are stabilized with respect to those at low densities. However, in the true polymer-induced interaction the three- and more-body terms add a repulsive contribution such, that the attractive two-body contribution is almost completely compensated, $\Omega^{*} / V \ll 1$. Hence, the relative stability of the fluid and solid phase upon increasing the polymer fugacity does hardly change, and this is reflected in the almost constant densities along the fluid-solid coexistence line.

\section{B. Scaled-particle theory and thermodynamic perturbation theory}

For the AO-model, scaled-particle theory ${ }^{43,27,26}$ provides an approximate expression for the free volume in the $(z=0)$ hard-sphere reference system. This expression is given by Eq. (16). In Fig. 7 we have plotted, along with the simulation results for the AO-model, the results predicted by scaledparticle theory. In the fluid phase the theoretical results compare quite well with the simulation results. This is consistent with the fact that scaled-particle theory gives an accurate prediction for the equation-of-state of a hard-sphere fluid. However, in the solid range the scaled-particle prediction strongly overestimates the free volume (not visible on the scale used in Fig. 7). This is not surprising as the scaledparticle expression was constructed to describe the fluid phase. As the free volume in the solid range is relatively small, in particle for "long" polymers, this will affect the thermodynamic properties only at high values of the polymer fugacity.

Once the free volume in the hard-sphere reference system is known, one can apply first-order thermodynamic perturbation theory [Eq. (15)] to compute the thermodynamic properties. In most theories of colloid-polymer mixtures the free volume is determined either (1) by approximating the polymer-induced interaction by a two-body interaction and using known results for the local structure of the hard sphere fluid and solid ${ }^{13.14,20,24}$ or (2) using the expression provided by scaled-particle theory. ${ }^{27,26}$ In order to test the validity of first-order thermodynamic perturbation theory we applied it to the $z=0$ simulation results for the free volume in the lattice-polymer model and the AO-model (see Tables II and
III). These results were inserted in the equations for the pressure (12) and the chemical potential (14). Subsequently the coexisting state points were determined numerically. The resulting phase diagrams are plotted as dotted lines, along with the full simulation results (drawn lines), in Fig. 8 (latticepolymer model; $l=10$ ), Fig. 9 (lattice polymer model; $l=50$ and $l=100$ ), and Fig. 10 (AO model; $\sigma_{\mathrm{pol}}=5.2075$ and $\left.\sigma_{\mathrm{pol}}=7.3104\right)$.

For the "short" $l=10$ polymers, the phase diagram obtained by thermodynamic perturbation theory compares quite well with the simulation results, except for the region with relatively high fugacities. In this region the theoretical prediction for the coexisting fluid density lies significantly above the simulation result. For the lattice polymer models with the "long" $l=50$ and $l=100$ polymers and the AOmodel, the location of the fluid-solid phase boundary and the triple point obtained by thermodynamic perturbation theory agrees very well with the simulation results. However the vapor-liquid phase boundaries differ significantly; the perturbation theory predicts a significant lower value for the critical fugacity. For the regions where the theory differs from the simulation results it is to be expected that the local structure of the colloidal fluid differs significantly from the hard-sphere fluid used as a reference in the perturbation calculations. Hence, it should not be surprising that in these regions the perturbation theory fails.

Assuming a linear dependence of the ratio of the triple and critical fugacity, we estimate from the results of the perturbation calculations that the liquid phase disappears for polymer-colloid size ratios $2 R_{g} / \sigma_{\text {col }} \leqslant 0.44$, for the latticepolymer model, and $\sigma_{\mathrm{pol}} / \sigma_{\mathrm{col}} \leqslant 0.41$, for the AO-model. The latter value is somewhat higher than the value $\sigma_{\mathrm{pol}} / \sigma_{\mathrm{col}} \leqslant 0.32$, obtained by a combination of scaled-particle theory and thermodynamic perturbation theory. ${ }^{26,27}$ This difference could be attributed the inaccuracy of the scaledparticle theory for the high-density fluid range, and the solid range.

In conclusion, the comparison shows that scaled-particle theory provides a fairly accurate prediction of the free volume in the AO-model, for densities in the fluid range. However, it fails for densities in the solid range. Moreover, we have seen that first-order perturbation theory yields a fairly accurate prediction for the phase diagram at high colloid densities, but cannot account for the phase behavior in regions with moderate and low colloid densities, including the region near the critical point.

\section{SUMMARY}

By computer simulation we studied a simple model for a colloid (hard spheres, diameter $\sigma_{\mathrm{col}}$ ) dispersed in a polymer solution (ideal lattice chains, radius-of-gyration $R_{g}$ ). Using a recently developed numerical scheme we were able perform Monte Carlo simulations at constant polymer fugacity, taking into account the polymer-induced interaction exactly.

For various polymer lengths we computed the equationof-state, i.e., the pressure and the colloid chemical potential as function of the colloid density and polymer fugacity. Using these results we determined the phase diagram. For "short" polymers the phase diagram is of the fluid-solid 
type, whereas for the "long" polymers the colloidal fluid phase separates into a low density vapor and a high density liquid phasc. Hence, our simulations are consistent with the experimental evidence ${ }^{12,14,17}$ that for the existence of a colloidal liquid phase sufficiently "long" polymers are required. More generally, our results confirm (see, e.g., Refs. 13, 24, 26) that in a system interacting via a steep (hard-sphere-like) repulsion with an attractive tail, a stable liquid phase can only occur if the ratio of the range of the attractive and repulsive parts of the interaction is sufficiently large. From the present results we can estimate that the minimal sizeratio $2 R_{g} / \sigma_{\text {col }}$ necessary to induce a colloidal vapor-liquid coexistence in the lattice-polymer model for a colloidpolymer mixture is 0.45 . For a hard-sphere-like colloid dispersed in an aqueous polymer solution this size-ratio has been determined experimentally to be $0.2 .^{17}$ The difference should be attributed to the fact that in this work we considered simplified models, that cannot be compared directly with experimental systems.

For comparison we have performed simulations of the AO model, that is similar to the lattice-polymer model, except for the fact that the polymers are represented by interpenetrable spheres. Only for high colloid densities the com parison of the free volumes of both models showed a discrepancy. At these densities the free volume in the AOmodel turned out to be significantly less than in the corresponding lattice-polymer model. Nevertheless, for the range of polymer fugacities considered, the computed phase diagram of AO-models reproduces most or the properties of the lattice-polymer model quite well. However, it should be stressed that the present study only considers systems where the colloidal particles are larger than the radius-of-gyration of the polymers. It is to be expected that in the opposite case the deformability of the polymers will play an increasingly important role, in particular at high colloid densities.

We have tested the validity of various assumption made in theories of colloid-polymer mixtures by a quantitative comparison of theoretical results with our simulation results. First of all, we have found that for all but the shortest polymers the polymer-induced attraction is not pairwise additive in the colloidal coordinates. The three- and more-body terms add a significant repulsive contribution. Neglecting the many-body terms does lead not only a drastic change in the local structure of the colloidal fluid, ${ }^{25}$ but also to a significant shift of the critical and triple point to lower fugacities, and a decrease in the range of fugacities with stable liquid phase. This comparison suggests that the assumption of pairwise additivity, used in many of the theoretical descriptions, is an oversimplification.

Second, we have found that thermodynamic perturbation theory yields a fairly accurate prediction for the location of the phase boundaries obtained at high colloidal densities, but fails for lower values of the colloid density, including the region near the critical point. Hence, in order to describe the thermodynamic properties at moderate and low fluid densities, an improvement of existing theoretical methods is required.

In this work we have ignored polymer-polymer interactions. It is to be expected that such interactions will affect the phase behavior at high polymer fugacities. Using an extension of the present scheme, simulations of such systems can be performed in a mean-field approximation. ${ }^{44}$ Alternatively, one can resort to a direct simulation of a mixture of colloids and self-avoiding polymers. The latter approach is, however, computationally demanding and has, to our knowledge, not yet been used to study polymer-colloid phase diagrams.

\section{ACKNOWLEDGMENTS}

We thank A. Vrij, H. N. W. Lekkerkerker, and B. M. Mulder for useful suggestions and discussions. This work is part of the research program of the Stichting voor Fundamenteel Onderzoek der Materie (Foundation for Fundamental Research of Matter) and was made possible by financial support from the Nederlandse Organisatie voor Wetenschappelijk Onderzoek (Netherlands Organization for the Advancement of Research), and the Dutch Ministry of Economic Affairs within the framework of the Innovatief Onderzoeksprogramma Polymeer-composieten en Bijzondere Polymeren (Innovative Research Program Polymercomposites and Special Polymers). Computer time on the CRAY-YMP at SARA, was made available through support of the Stichting Computer Faciliteiten (Foundation for $\mathrm{Na}$ tional Computing Facilities).

'B. R. Monoghan and H. L. White, J. Gen. Physiol. 19, 715 (1935).

${ }^{2}$ C. Bondy, Trans. Faraday Soc. 35, 1093 (1939).

${ }^{3}$ S. Asakura and F. Oosawa, J. Chem. Phys. 22, 1255 (1954).

${ }^{4}$ A. Vrij, Pure Appl. Chem. 48, 471 (1976).

${ }^{5}$ C. Cowell, F. K. R. Li-In-On, and B. Vincent, J. Chem. Soc. Faraday Trans. I 74, 337 (1978).

${ }^{6} \mathrm{H}$. de Hek and A. Vrij, J. Colloid Interface Sci. 70, 592 (1979).

${ }^{7}$ B. Vincent, P. F. Luckham, and F. A. Wait, J. Colloid Interface Sci. 73, 508 (1980).

${ }^{8}$ H. de Hek and A. Vrij, J. Colloid Interface Sci. 84, 409 (1981).

${ }^{9} \mathrm{~J}$. Clarke and B. Vincent, J. Colloid Interface Sci. 82, 208 (1981).

${ }^{10}$ F. K. R. Li-In-On, B. Vincent, and F. Waite, ACS Symp. Ser. 9, 309 (1983).

${ }^{11}$ B. Vincent, J. Clarke, and K. G. Bernett, Colloids Surf. 117, 51 (1986).

${ }^{12}$ P. R. Sperry, J. Colloid Interface Sci. 99, 97 (1984)

${ }^{13}$ A. P. Gast, W. B. Russel, and C. K. Hall, J. Colloid Interface Sci. 1, 161 (1986).

${ }^{14}$ B. Vincent, J. Edwards, S. Emmett, and R. Groot, Colloid Surf. 31, 267 (1988).

${ }^{15}$ P. Tong, T. A. Witten, J. S. Huang, and L. J. Fetters, J. Phys. France 51, $2813(1990)$

${ }^{16}$ W. C. K. Poon, J. S. Selfe, M. B. Robertson, S. M. Ilett, A. D. Pirie, and P. N. Pusey, J. Phys. II France 3, 1075 (1993).

${ }^{17}$ R. L. Calderon, J. Bibette, and J. Biais, Europhys. Lett. 23, 653 (1993).

${ }^{18}$ G. J. Fleer, J. H. M. H. Scheutjens, and B. Vincent, ACS. Symp. Ser. 240, 245 (1984).

${ }^{19}$ J. F. Joanny, L. Leibler, and P. G. de Gennes, J. Polymer Sci. Polymer. Phys. Ed. 17, 1073 (1979).

${ }^{20}$ A. P. Gast, C. K. Hall, and W. B. Russel, Faraday Discuss. Chem. Soc. 76, 189 (1983).

${ }^{21}$ E. Canessa, M. J. Grimson, and M. Silbert, Mol. Phys. 67, 1153 (1989).

${ }^{22}$ M. R. Shaw and D. Thirumalai, Phys. Rev. A 44, R4797 (1991).

${ }^{23}$ A. Yethiraj, C. K. Hall, and R. Dickman, J. Colloid Interface Sci. 151, 102 (1991).

${ }^{24}$ A. P. Gast, C. K. Hall, and W. B. Russel, J. Colloid Interface Sci, 96, 251 (1983).

${ }^{25}$ E. J. Meijer and D. Frenkel, Phys. Rev. Lett. 67, 1110 (1991).

${ }^{26}$ H. N. W. Lekkerkerker, W. C-K. Poon, P. N. Pusey, A. Stroobants, and P. B. Warren, Europhys. Lett. 20, 559 (1992).

${ }^{27}$ H. N. W. Lekkerkerker, Colloid Surf. 51, 419 (1991).

${ }^{28}$ N. F. Carnahan and K. E. Starling, J. Chem. Phys. 51, 635 (1969).

${ }^{29}$ W. G. Hoover and F. H. Ree, J. Chem. Phys. 49, 3609 (1968). 
${ }^{30}$ K. R. Hall, J. Chem. Phys. 57, 2252 (1972).

${ }^{31}$ B. J. Alder, W. G. Hoover, and D. A. Young, J. Chem. Phys. 49, 3688 (1968).

${ }^{32}$ R. Zwanzig, J, Chem. Phys. 22, 1420 (1954).

${ }^{33} \mathrm{H}$. Reiss, H. Frisch, and J. Lebowitz, J. Chem. Phys. 31, 369 (1959).

${ }^{34}$ J. L. Lebowitz. E. Helfand, and E. Praestgaard, J. Chem. Phys. 43, 774 (1965).

${ }^{35}$ D. Frenkel, J. Phys. Condensed Matter 2, SA265 (1990).

${ }^{36}$ P. Flory, Statistical Mechanics of Chain Molecules (Carl Hanser Verlag. Munich, 1989).

${ }^{37}$ D. A. Kofke, Mol. Phys. 78, 1331 (1993).
${ }^{38}$ D. A. Kofke, J. Chem. Phys. 98, 4149 (1993).

${ }^{39}$ W. H. Press, B. P. Flannery, S. A. Teukolsky, and W. T. Vetterling, Numerical Recipes (Cambridge University, Cambridge, 1986).

${ }^{40}$ B. Widom, J. Chem. Phys. 39, 2808 (1963).

${ }^{41}$ J. S. Rowlinson and F. L. Swinton, Liquids and Liquid Mixtures, 3rd ed. (Buttersworth, London, 1982).

${ }^{42} \mathrm{~J}$. S. Rowlinson and B. Widom, Molecular Theory of Capillarity (Clarendon, Oxford, 1982).

${ }^{43}$ J. L. Lebowitz and J. K. Percus, Phys. Rev. 144, 251 (1966).

${ }^{44}$ E. J. Meijer and D. Frenkel (to be published). 